\title{
50th ANNUAL SASKATCHEWAN CHRISTMAS BIRD COUNT - 1991
}

Compiled by WAYNE C. HARRIS, Box 414, Raymore, Saskatchewan. SOA 3J0

In this the fiftieth year of the Christmas Bird Counts being published in the Blue Jay (it is also anniversary number 50 for both the Blue Jay and the Saskatchewan Natural History Society) it seems appropriate that this is the best year ever for the counts. It has been a long time since five new species were added to the list in one year and never before have there been so many people on so many counts. This was all enhanced by the nicest weather we have had for a long time for the entire count period (14 December - 2 January). The only drawback that became very obvious through the compilation effort was that although nice weather enhances the quantity of data, it would be, from a compiler's perspective, much easier to complete the compilation if the weather had been $-50^{\circ}$ and the winds blowing continuously. At least I think that compiling counts when none were done would be easier than the 99 that were received this year!

\section{Weather and Coverage}

Table 1 summarizes the absolutely beautiful weather conditions we endured to carry out the 1991 count. Many days were above freezing, the winds were generally light and the skies clear. There was virtually no snow fall during the count period and no blizzards to be endured. One has to really search to find a minimum temperature below $-18^{\circ} \mathrm{C}\left(0^{\circ} \mathrm{F}\right)$.

With the warm weather more people (667) participated in more counts (99) than in any of the past years (Count areas and Participants follows). Formerly the most people to participate was 611 in 1987 while the maximum number of counts was 84 in 1988. Over 1200 hours were spent watching birds this Christmas!

\section{The bird counts}

There were 96 species and 2 forms reported from the 99 counts this year and all but 4 of the species were recorded on count day. A total of about 74,000 birds were seen, about three quarters the number reported last year. Some of the largest decreases in numbers were for Mallard down about 14,000, Snow Buntings 7000, Horned Lark -5700 and Lapland Longspur -4200 . These reductions do not necessarily reflect actual population declines but rather are more likely a result of shifting wintering areas. The precount weather which was in late fall much colder and with heavier snowfalls forced many of these birds to move to more hospitable conditions and once further south they are not likely to return until spring. This is particularly true of the Horned Lark, longspur and Mallard. With the drop in Snow Bunting numbers the House Sparrow became the most abundant species (18,957 individuals) and followed only the Black-billed Magpie (91 of 99 counts), Black-capped Chickadee (91 counts), and Common Raven (90 counts) as the most frequently recorded species, with records from 88 locations.

Saskatoon again led with greatest variety of species with 42 followed closely by Squaw Rapids with 41, Fort Walsh and Fort Qu'appelle with 39 species and Weyburn with 38 . Other counts with over 30 species include Raymore with 35, Glentworth with 32 , and Regina, Indian Head and Hudson Bay, all with 31 .

\section{New Species}

Five species were seen for the first time on a Saskatchewan Christmas bird count raising the overall total to 158 
species. The first of these new species was a Yellow-billed Loon at Gardiner Dam. This bird had been present for some time and has been seen by a considerable number of people. The Yellow-billed Loon normally winters in coastal areas and inland records anywhere are rare.

The second new species was a Redheaded Woodpecker at White Bear Lake in the Moose Mountain area. Although this species is quite rare even in the summer in Saskatchewan there are several Manitoba winter records. A Black-headed Grosbeak was found on the Moose Jaw count. This species normally would be in the tropics during the winter months. A Savannah Sparrow visiting a feeder in Saskatoon was the fourth new species for the count. The last new species was the Chestnut-collared Longspur photographed on the Govenlock count. Although this is a first record of this species for a Saskatchewan $\mathrm{CBC}$ and I think for Canada it is probably the least surprising considering the abundance of this species in this area during the summer.

\section{Rare species}

For the second year in a row a Harlequin Duck was reported, this time from Fort Qu'appelle. The Turkey Vultures at Grayson and the Fox Sparrow at Saskatoon were the third records. The Whitecrowned Sparrow at Weyburn was only the fourth record. The Squaw Rapids' Common Loon was the sixth record.

\section{Population trends}

Some of the more outstanding declines were mentioned earlier but several other species declined to a similar degree even though the numbers were not as spectacular. Short-eared Owls were reported from only 7 counts and only Glentworth had more than one. The fact that owls in general were less common in the farmland regions may reflect low prey populations. This may also apply to the low numbers of Rough-legged Hawks. Both Brown Creeper and Golden- crowned Kinglets were undoubtedly reduced by the early cold weather.

On the positive side was the increase in numbers of the members of the blackbird family. The early heavy snowfall may have forced these species to seek food in livestock feedlots and with the food source available to them many decided to stay instead of continuing their migration southward. The most notable of these was the Western Meadowlark which, prior to this year, had been found only 20 times on counts and this year there were 11 records.

One other influx deserves mention. Northern Hawk Owls were surprisingly common along the forest fringe. Previously there had been 40 counts with only 33 individuals on count day. This year there were 18 counts with 32 count day individuals, doubling the previous 49 years' total in just one year! Great Gray Owls also had large increases over past years.

\section{Count Areas and Participants}

(Names of compilers are in italics, number of observers in parentheses at end of list of names.)

1. ABERNETHY-KATEPWA. Bryan Bittner, Ron Bittner, Phyliss Bordass, Del Cairns, Ron Hooper, Bob Kreba, Marj Leader, Steve Lucyk, Joy McKen, Norman McKen, Wendy Paquin, Lorne Rowell, Keith Stephens, Wanda Stueck. (14)

2. ARMIT. Les Baker, Anne Harris, Wayne Harris, Donald Hooper, William Zak. (5)

3. BANGOR. Jean Hilton, Minnie Hughes, Bill and Sheila Popowich. (4)

4. BATTLEFORD. Jean Anderson, MaryJean and Ron Dewald, Barry Craig. (4)

\section{BETHUNE. Doug and Vera Laing. (2)}

6. BIGGAR. Roger Assailly, Guy Wapple, Robert Wapple, Sandra Wapple. (4)

7. BIG MUDDY LAKE. Martin Bailey, Carol Bjorklund. (2) 
8. BIG RIVER. Glenn Honig. (1)

9. BIRCH HILLS. Moe Mareschal, Marg Mareschal, Don Weidl. (3)

10. BORDEN. Wayne Harris. (1)

11. BRIGHTWATER RESERVOIR. A.R. Smith. (1)

12. BROADVIEW. Dave Chaskavich, Don Weidl. (2)

13. BROMHEAD. Martin Bailey, Carol Bjorklund. (2)

14. CANDLELAKE. Burke Korol, Harv Lane. (2)

15. CHOICELAND-GRONLID. Wayne Harris. (1)

16. CLARK'S CROSSING. Carol Blenkin, Sandra Flood, Al Hartley, Erling Larsen, Grethe Larsen, Kevin Moore, Menno Nickel, Ben Rosser, Hilda Voth, Michael Williams. (10)

17. CONQUEST-OUTLOOK. Jill Forrester, A.R. Smith. (2)

18. CRAVEN. Martin Bailey, Margaret Belcher, Al Binnie, Betty Binnie, Carol Bjorklund, Phyllis Ilsley, Ferne Lawrence, Milow Worel. (8)

19. CROOKED LAKE (A). Doug Boivin, Don Weidl, Tony Weidl. (3)

20. CROOKED LAKE (B). Hazel and Vern Bjorgen, Bill and Mayta Livesay, Dorothy Skene, Ed Skene. (6)

21. CROOKED RIVER. Anne Harris, Valeri Harris, Wayne Harris. (3)

22. DALMENY. Loyd Sperling. (1)

23. DILKE. Margaret Belcher, Myrla Holland, Ken McArton, George Metz. (4)

24. DUVAL. George Herber, Mervin Hey, lain Richardson, Lloyd Saul. (4)

25. EDAM. Marg Terpstra, Tom Terpstra. (2)

26. ENDEAVOUR. Norman Harris. (1)

27. FENTON. Carman Dodge. (1)

28. FORT QU'APPELLE. John Banick, Ethel Cockwill, Ann Davies, Frank Davies, Evelyn Fleming, Doreen Harman, Ronald Hooper, Naomi Jardine, Cal Keller, Bob Kreba, Lois Lamontagne, Maurice Lindgren, Don McDougall, Allan Mlazgar, Paul Paquin, Lorne Rowell, Lloyd Talbot. (17)
29. FORT WALSH. Martin Bailey, Carol Bjorklund, Dixie Black, Anemone Harris, Wayne Harris, Burke Korol, Wilkes Parsonage, Guy Wapple, Robert Wapple. (9)

30. GARDINER DAM. Martin Bailey, Carol Bjorklund, Anemone Harris, Valeri Harris, Wayne Harris, Burke Korol, Guy Wapple, Robert Wapple. (8)

\section{GLENTWORTH. Wayne Harris. (1)}

32. GOOD SPIRIT LAKE. Bill Anaka, Joyce Anaka, Julia Wiwchar. (3)

33. GOVENLOCK. Martin Bailey, Carol Bjorklund, Anne Harris, Valeri Harris, Wayne Harris, Burke Korol, Guy Wapple, Robert Wapple. (8)

34. GRASSLANDS NATIONAL PARK. Anne Harris, Valeri Harris, Wayne Harris, Jacques Saquet. (4)

35. GRAYSON. Charles Helm, Karl Zimmer. (2)

36. GRENFELL. Jim Elliot. (1)

37. HEPBURN. Phyllis Siemens. (1)

38. HUDSON BAY. Moe Alain, Les Baker, Pat Beaulieu, Gerald Coates, Anne Harris, Wayne Harris, Les Hayes, Donald Hooper, Fumi Torigai, Taeko Torigai, Tsetse Torigai, Gloria Stang. (12)

39. HUMBOLDT. Ed Brockmeyer, Warren Hoffman, Mike Volk. (3)

40. INDIAN HEAD. Carol Beaulieu, Denise Beaulieu, Vic Beaulieu, Doug Derksen, Irv Escott, Dave Gehl, Roger Grey, Mavis Grey, Gordon Howe, John Kort, Linda Kort, Mary Kort, Peter Kort, Don Longeau, Fran Maddia, Erin Neill, Doug Nerby, Helen Nerby, Dora Nichols, Norine Nichols, Lou Osiowy, Lorne Scott, Chris Skinner, Fred Skinner, Linda Skinner, Charlie Thompson, Ruby Thompson, Anne Willerth, Gordon Willerth, Jackie Willoughby. (30)

41. KAMSACK. John Barisoff, George and Mary Bernhard, Agnes Betz, Sally Bishop, Mable Buceuk, Lindee Dewores, Doris Franklin, Bill Koreliuk, W.J.C. May, Ann McIvor, Dallas and Jesse Rezansoff, Isabel Ritchie, Wally Sasyniuk, John Solomon, Elenor Sookocheff, Ted and Thoreen Wallace, Ann and Gordon Wlasichuk. (22) 
42. KELVINGTON. Pat Finnie, Chet Sloan, Diane Sloan, Marguerite Sloan. (4)

43. KENASTON. Doug Beckie, Lawrence Beckie, Lori Cooper. (3)

44. KILWINNING. E.A. Driver, M.E. Driver. (2)

45. KINDERSLEY. Gerry and Michael Essar. (2)

46. KINLOCH. Don Forbes, Doreen Forbes, Alice Rodenberg, Wilf Rodenberg. (4)

47. KUTAWAGAN LAKE. Wayne Harris, Sheila Lamont. (2)

48. LA RONGE. Cheryl Paul, Jim Paul, Lorie Ann Paul. (3)

49. LAST MOUNTAIN LAKE WILDLIFE MANAGEMENT UNIT. Anne Harris, Valeri Harris, Wayne Harris, Sheila Lamont. (4)

\section{LEADER. Daisy D. Meyers. (1)}

\section{LIVELONG. Sarah Pavka. (1)}

52. LOVE-TORCHRIVER. Dot and Ed Bennett, Bert Dalziel, Duke Dalziel, Joan Dalziel, Kari Dalziel, Sara Dalziel, Betty Donovan, Bruce Donovan, Mildred Long, Bill Matthews, Lynn Matthews, Irene Vivian. (13)

53. LUSELAND. Estelle Finley, Kim Finley, Bill Frey, Bill Holton, Donna Holton, Travis Holton, Virginia Holton, Bev Honeker, Brent Honeker, Dustin Honeker, Levi Honeker, Alexis Martfeld, Katrina Martfeld, Travis Martfeld, Wyatt Martfeld, Joe Rozlein. (16)

54. MACDOWALL. Myron Barton, Marie Best, Lon Borgerson, Val Drummond, Bill Gibbs, Norman Kernohan, Gladys Kernohan, Henry Neudorf, Sylvia Neudorf, Eileen Wilker, Harry Wilker. (11)

55. MATADOR. Cindy Romo, J.T. Romo. (2)

56. MEADOW LAKE. Pat Dunk, Deb Eisenbut, Betty Ann Golly, Byron Golly, Stuart Golly, Tyler Golly, Bob Wilson, George Wood, Gladys Wood. (9)

\section{MELFORT. Frieda Markland. (1)}

58. MELVILLE. Marion MacLean, Bernie and Marilyn Sheppard, Mildred Wotherspoon, Ross Wotherspoon. (5)

59. MOOSE JAW. Mac Aldred, Andy and Vi Anderson, Edith Bell, Doug and Helen Brunsdon, Carol Clelland, Muriel Dormer, Doug and Pat Francis, Ed and Tristan Fredeen, George Grigg, Al Gurnsey Kerry Hanley, Eve King, Cy Knight, Leith Knight, Connie and Hugh Mclntyre, Nancy and Vivien Meikle, Pierre Morrisette, Gavina Reekie, Gus Sagal, Ed and Grace Walker. (27)

60. NAICAM. Eldor Jensen, Julie Jensen, Ron Jensen. (3)

61. NEELY LAKE. Dawn Billeter, Edwin Billeter, Shirley Billeter, Donald Hooper, Gordon Johnson, Pauline Lindenbach, Betty Salmond. (7)

62. NIPAWIN. Vi Budd, Joyce Christiansen, Doug Pegg, Helen Pegg, Doug Phillips, Shirley Phillips. (6)

63. PIKE LAKE. Mrs. Marcel Bernier, Carol Blenkin, Kent Brace, Muriel Carlson, Melanie Elliott, Mary Gilliland, Bernie Gollop, Mike Gollop, Debbie Lashta, Frank McDougall, Kevin Moore, Doug and Marguerite Schneider, Aire Van Duyvendyk, Michael Williams, Jim Wood, Lois Wooding. (17)

64. PORCUPINE PLAIN. Les Baker, David Black, Frank Gabriel, Donald Hooper, Keith Larwood, Pat Larwood, Paula Remple. (7)

\section{PREECEVILLE. JimandMary Grimsrud. (2)}

66. PRINCE ALBERT (A). Elsie Morton. (1)

67. PRINCE ALBERT (B). Carol Beck, Ron Beck, John Burt, Pam Burt, Evelyn Chapuis, Carman Dodge, Keith Dodge, Joe Graumans, Hamilton Greenwood, George Huczek, Ted Snow. (11)

68. PRINCE ALBERT NATIONAL PARK. Marilyn Anions, Susan Carr, Chris Clinton, Eileen Clinton, Michael Fitzsimmons, Teri Jackson, Leanne Martin, Brad Muir, Steve Oates, Grant Peregoodoff, Adam Pidwerbeski, Julie Stocki, Colleen Watson, Fred Weil, Mike Wynn. (15) 
69. QU'APPELLE VALLEY DAM. Al Hartley, Stan Shadick, Michael Williams, Jim Wood. (4)

70. RAYMORE. Anne Harris, Valeri Harris, Wayne Harris, Sheila Lamont. (4)

71. READ LAKE. Daryl Johannesen, Don Weidl. (2)

72. REGINA. John Allen, Sandy Ayer, Jessie Bailey, Martin Bailey, Margaret Belcher, Carol Bjorklund, Lionel Bonneville, Stephane Bonneville, Ryan Csada, Joanne Edwards, Kathy Ferguson, Kay Ferguson, Malcolm Hayes, Wilbur Heinrich, Dale Hjertaas, Estelle Hjertaas, Kerry Hjertaas, Paule Hjertaas, Phyllis Ilsley, Matthew James, Nicholas James, Paul James, Bob Kreba, Wee Lee, Christine MacDonald, Lauren Mang, Scott McKenzie, Yvonne McKenzie, Colin Morrell, Judy Morrell, Rick Morrell, Ron Myers, Mary Ann Nelson, Gwen Norelius, John Pollock, Brian Rainey, Gillian Richardson, Trevor Richardson, Dean Richert, Miriam Richert, Lyle Saigeon, Diane Secoy, Frank Switzer, lan Switzer, Kathy Winston. (45)

73. ROUND LAKE. Ross Baker, Doug Francis, Pat Francis, Joe and Laura Grimeau, Olga MacDonald, Harry and Rita Minovitch, Gary Tinnish. (9)

74. SASKATOON. Norma Allen, Juhachi Asai, Kengo Asai, Mika Asai, Rika Asai, Sumiko Asai, Yuka Asai, lan Bennett, Garth Besant, Jeff Besant, Joyce Besant, Maria Besant, Robert Besant, Bernard Bisha, Carol Blenkin, Barbara Brokx, Emile Brokx, Muriel Carlson, Bill Cates, Donna Cates, Paul Coutu, Vi Coutu, Amanda Dalglish, Eileen Dalglish, Fred Doner, Ann Dzus, Eric Ehman, Jeffrey Ehman, Helen Fast, Martin Gerard, Silvia Gerard, Colleen Gerwing, Bernadette Gilliland, Marshall Gilliland, Mary Gilliland, Sean Gilliland, Bob Godwin, Bernie Gollop, M.F. Gollop, John Hanbidge, Peter Hankowich, Ramona Harms, Martha Ann Helgerson, Adam Houston, David Houston, Margaret Houston, Mary Houston, Stuart Houston, Don Johnston, Janice Laliberte, Debbie Lashta, Don McRobbie, Jo McRobbie, Kathy Meeres, Cathryn Miller, David Miller, Quentin
Miller, Sue Mitten, Kevin Moore, Lisa Napier, Menno Nickel, Brian Olson, Ben Rosser, Lynn Rowland, Aleta Silverhorn, Philip Taylor, Ivor Thokle, David Thorpe, Jeff Turple, Hilda Voth, Heather Wagg, Jim Wedgwood, Michael Williams, Jim Wood, Lois Wooding. (75)

\section{SCOTT. Dale Booth, Guy Wapple. (2)}

76. SIMPSON. Thomas Harper. (1)

77. SKULL CREEK. Frank Belhof, James Bennetto, Ray Bennetto, Doris Bircham, Ralph Bircham, Bob Eccleston, Phyllis Flaig, Corey Wasilow, Harvey Wasilow, Brad Wolfater, Robin Wolfater, Ron Wolfater. (12)

78. SOMME. Sharon Birch, David Black, Louise Butterfield, Donald Hooper, Margaret Hooper, Alex Kirk. (6)

79. SPALDING. Velma Spizawka, William Spizawka. (2)

80. SPINNEY HILL. Ed Driver, Philip Taylor. (2)

81. SPRING VALLEY. Allan Bogdan, Bryan Bogdan, Flossie Bogdan, Larry Bogdan, Nick Bogdan, Dean Goin. (6)

\section{SPRUCE HOME. Sharon and Tom Dice. (2)}

83. SQUAW RAPIDS. Anne Harris, Valeri Harris, Wayne Harris, Guy Wapple. Robert Wapple. (5)

84. SWIFT CURRENT. Jim Beaty, Meryl Campion, Ron Jensen, Velma Kinsman, Max Mirau, Anne Sakata, Ron Sakata, D. Thoreson, Kae Waters, John Weston, Pearl Weston, Betty Welgan, Ron Welgan. (13)

\section{TISDALE. Joyce Mohr. (1)}

\section{TOMPKINS. Raymond OIson. (1)}

87. VAL MARIE. Aaron Bakus, Tobert Bakus, Tyler Cherpin, Nicky Coté, Roy Coté, Logan Grant, Lynn Grant, Judy Facette, Curtis Franklin, Jamie Franklin, Ben McDonald, Donald McDonald, Cheryl Penny, Jacques Saquet, JeanClaude Saquet, Michelle Saquet. (16) 
Table 1. COUNT WEATHER CONDITIONS ( $T^{\circ} \mathrm{C}$, Wind $\mathrm{Kmph}$, Snow $\mathrm{Cm}$ )

\begin{tabular}{|c|c|c|c|c|c|c|c|c|}
\hline COUNT NAME & $\begin{array}{l}\text { Min } \\
T^{\circ} \mathrm{C}\end{array}$ & $\begin{array}{l}\text { Max. } \\
T^{\circ} \mathrm{C}\end{array}$ & $\begin{array}{l}\text { Min. } \\
\text { Wind }\end{array}$ & $\begin{array}{l}\text { Max. } \\
\text { Wind }\end{array}$ & $\begin{array}{l}\text { Min. } \\
\text { Snow }\end{array}$ & $\begin{array}{l}\text { Max. } \\
\text { Snow }\end{array}$ & Sky A.M. & Sky P.M. \\
\hline 1. ABERNETHY-KATEPWA LAKE & -8 & +3 & 30 & 40 & 15 & 30 & mostly cloudy & mostly cloudy \\
\hline 2. ARMIT & -10 & 0 & 0 & 35 & 30 & 40 & partly cloudy & clear \\
\hline 3. BANGOR & -12 & -4 & 15 & 20 & 40 & 50 & mostly cloudy & clear \\
\hline 4. BATTLEFORD & -10 & -5 & 10 & 15 & & 5 & & \\
\hline 5. BETHUNE & -6 & -3 & 0 & 10 & 15 & 20 & & clear \\
\hline 6. BIGGAR & -6 & -2 & 20 & 30 & 10 & 30 & mostly cloudy & partly cloudy \\
\hline 7. BIG MUDDY LAKE & -1 & -1 & 0 & 5 & 0 & 2 & heavy fog & clear \\
\hline 8. BIG RIVER & -10 & -5 & 0 & 5 & 25 & 34 & mostly cloudy & mostly cloudy \\
\hline 9. BIRCH HILLS & 0 & +2 & 15 & 30 & 10 & 40 & clear & clear \\
\hline 10. BORDEN & -5 & -3 & 0 & 15 & 25 & 30 & overcast & clear \\
\hline 11. BRIGHTWATER RESERVOIR & -10 & -5 & 0 & 20 & 10 & 20 & clear & clear \\
\hline 12. BROADVIEW & -4 & +1 & 10 & 26 & 20 & 30 & clear & clear \\
\hline 13. BROMHEAD & -8 & -1 & 10 & 20 & 0 & 2 & partly cloudy & partly cloudy \\
\hline 14. CANDLE LAKE & -15 & -10 & 0 & 5 & 10 & 40 & mostly cloudy & mostly cloudy \\
\hline 15. CHOICELAND-GRONLID & -18 & -6 & 0 & 35 & 30 & 50 & clear & clear \\
\hline 16. CLARK'S CROSSING & -17 & -10 & 0 & 10 & 10 & 60 & clear & mostly cloudy \\
\hline 17. CONQUEST-OUTLOOK & -10 & -5 & 0 & 10 & 5 & 15 & clear & clear \\
\hline 18. CRAVEN & -13 & -6 & & 22 & & 15 & clear & clear \\
\hline 19. CROOKED LAKE(A) & 0 & +1 & 15 & 20 & 25 & 30 & clear & clear \\
\hline 20. CROOKED LAKE(B) & -3 & -2 & 2 & 10 & 35 & 45 & & overcast \\
\hline 21. CROOKED RIVER & -15 & -10 & 0 & 10 & 30 & 50 & partly cloudy & partly cloudy \\
\hline 22. DALMENY & -13 & -10 & 10 & 19 & 10 & 50 & clear & partly cloudy \\
\hline 23. DILKE & -6 & -3 & 15 & 30 & 0 & 20 & clear & clear \\
\hline 24. DUVAL & -10 & -4 & 0 & 7 & 0 & 30 & clear & clear \\
\hline 25. EDAM & -10 & -5 & 15 & 20 & 30 & 40 & clear & clear \\
\hline 26. ENDEAVOUR & -18 & +1 & 0 & 5 & 14 & 17 & partly cloudy & clear \\
\hline 27. FENTON & -16 & -5 & 0 & 6 & 5 & 25 & clear & mostly cloudy \\
\hline 28. FORT QU'APPELLE & -23 & -10 & 0 & 10 & 20 & 25 & & \\
\hline 29. FORT WALSH & -10 & -5 & 20 & 60 & 0 & 12 & partly cloudy & partly cloudy \\
\hline 30. GARDINER DAM & -3 & +1 & 20 & 50 & 0 & 20 & partly cloudy & clear \\
\hline 31. GLENTWORTH & -5 & +5 & 5 & 50 & 0 & 2 & clear & clear \\
\hline 32. GOOD SPIRIT LAKE & -3 & -1 & 20 & 25 & 15 & 34 & mostly cloudy & mostly cloudy \\
\hline 33. GOVENLOCK & -3 & +5 & 0 & 20 & 0 & 5 & clear & clear \\
\hline 34. GRASSLANDS NATIONAL FARK & -3 & +5 & 0 & 50 & 0 & 5 & clear & mostly clear \\
\hline 35. GRAYSON & -2 & +4 & & 40 & 5 & 25 & clear & clear \\
\hline 36. GRENFELL & -15 & -8 & & & 0 & 20 & & partly cloudy \\
\hline 37. HEPBURN & & & & & & & & \\
\hline 38. HUDSON BAY & 0 & +4 & 20 & 40 & 35 & 40 & mostly clear & mostly clear \\
\hline 39. HUMBOLDT & -10 & -5 & 5 & 20 & 10 & 40 & clear & clear \\
\hline 40. INDIAN HEAD & -10 & -5 & 0 & 5 & 10 & 30 & moderate fog & \\
\hline 41. KAMSACK & -14 & -9 & 10 & 15 & 75 & 125 & & \\
\hline 42. KELVINGTON & -26 & & 0 & 0 & & 75 & & clear \\
\hline 43. KENASTON & -8 & -4 & 0 & 15 & 0 & 15 & mostly clear & mostly clear \\
\hline 44. KILWINNING & -5 & -3 & 10 & 15 & 5 & 65 & clear & mostly clear \\
\hline 45. KINDERSLEY & -6 & -2 & 10 & 14 & 0 & 2 & mostly clear & mostly clear \\
\hline 46. KINLOCH & -5 & -8 & 0 & 10 & 45 & 60 & & \\
\hline 47. KUTAWAGAN LAKE & -8 & -2 & 0 & 20 & 20 & 40 & mostly clear & clear \\
\hline 48. LARONGE & -10 & -1 & 5 & 15 & 40 & 60 & clear & clear \\
\hline 49. LAST MOUNTAIN LAKE W.M.U. & -14 & -1 & 5 & 20 & 5 & 30 & clear & clear \\
\hline 50. LEADER & -10 & +2 & 0 & 5 & 0 & 3 & mostly clear & clear \\
\hline 51. LIVELONG & -15 & -5 & 0 & 0 & & 25 & mostly clear & mostly clear \\
\hline 52. LOVE-TORCH RIVER & -6 & -4 & 0 & 5 & 30 & 90 & overcast & overcast \\
\hline 53. LUSELAND & -6 & -1 & 0 & 5 & 22 & 49 & mostly clear & clear \\
\hline 54. MACDOWALL & 0 & +4 & 15 & 20 & 30 & 80 & mostly clear & partly cloudy \\
\hline 55. MATADOR & -2 & +2 & 0 & 10 & 0 & 10 & clear & clear \\
\hline 56. MEADOW LAKE & -6 & -2 & 0 & 0 & 24 & 120 & clear & clear \\
\hline 57. MELFORT & -16 & -1 & 0 & 5 & 60 & 110 & partly cloudy & mostly clear \\
\hline 58. MELVILLE & -10 & 0 & 0 & 0 & 20 & 100 & mostly clear & \\
\hline 59. MOOSE JAW & -11 & -1 & 8 & 12 & 3 & 18 & mostly clear & mostly clear \\
\hline 60. NAICAM & -5 & 0 & & 20 & 15 & 60 & clear & mostly clear \\
\hline 61. NEELY LAKE & -23 & -18 & 0 & 0 & 30 & 45 & clear & clear \\
\hline 62. NIPAWIN & -5 & -7 & & 5 & 10 & 50 & & mostly clear \\
\hline 63. PIKE LAKE & -2 & +3 & 15 & 30 & 20 & 50 & & \\
\hline 64. PORCUPINE PLAIN & -28 & -20 & 0 & 3 & 30 & 45 & m. clear, light snow & clear \\
\hline 65. PREECEVILLE & -18 & -6 & 0 & 5 & 40 & 65 & clear & \\
\hline 66. PRINCE ALBERT(A) & & & & & & & & \\
\hline 67. PRINCE ALBERT(B) & -5 & +4 & 5 & 20 & 5 & 25 & & \\
\hline 68. PRINCE ALBERT NATIONAL PARK & -10 & -15 & 0 & 10 & 30 & 37 & overcast & overcast \\
\hline 69. QU'APPELLE VALLEY DAM & -18 & -14 & 0 & 15 & 10 & 60 & p. cloudy, light snow & mostly clear \\
\hline 70. RAYMORE & -18 & -3 & 0 & 20 & 25 & 40 & clear & clear \\
\hline 71. READ LAKE & & -15 & 25 & 35 & 46 & 50 & partly cloudy & mostly cloudy \\
\hline 72. REGINA & -14 & -2 & & 25 & & 20 & clear & clear \\
\hline 73. ROUND LAKE & -10 & -5 & 0 & 5 & 3 & 20 & clear & clear \\
\hline 74. SASKATOON & -10 & -1 & 13 & 22 & & 26 & partly cloudy & mostly clear \\
\hline 75. ScoTT & -17 & -10 & 0 & 20 & 0 & 25 & clear & mostly clear \\
\hline 76. SIMPSON & -5 & -2 & 5 & 7 & 30 & 40 & clear & clear \\
\hline 77. SKULL CREEK & +4 & +5 & 40 & 45 & 0 & 0 & partly cloudy & partly cloudy \\
\hline 78. SOMME & -12 & 0 & 5 & 40 & 40 & 45 & partly cloudy & partly cloudy \\
\hline 79. SPALDING & -12 & -5 & 0 & 5 & 50 & 70 & clear & clear \\
\hline 80. SPINNEY HILL & -5 & -1 & 0 & 5 & 0 & 110 & & \\
\hline
\end{tabular}


Table 1. COUNT WEATHER CONDITIONS (Continued) ( $T^{\circ} \mathrm{C}$, Wind $\mathrm{Kmph}$, Snow $\mathrm{Cm}$ )

\begin{tabular}{|c|c|c|c|c|c|c|c|c|}
\hline COUNT NAME & $\begin{array}{l}\text { Min } \\
\mathrm{T}^{\circ} \mathrm{C}\end{array}$ & $\begin{array}{l}\text { Max. } \\
T^{\circ} \mathrm{C}\end{array}$ & $\begin{array}{l}\text { Min. } \\
\text { Wind }\end{array}$ & $\begin{array}{l}\text { Max. } \\
\text { Wind }\end{array}$ & $\begin{array}{l}\text { Min. } \\
\text { Snow }\end{array}$ & $\begin{array}{l}\text { Max. } \\
\text { Snow }\end{array}$ & Sky A.M. & Sky P.M. \\
\hline 81. SPRING VALLEY & -15 & -2 & 10 & 15 & 0 & 30 & partly cloudy & partly cloudy \\
\hline 82. SPRUCE HOME & -12 & -5 & 0 & 0 & 30 & 75 & overcast & overcast \\
\hline 83. SQUAW RAPIDS & -13 & -3 & 0 & 15 & 30 & 60 & mostly clear & mostly clear \\
\hline 84. SWIFT CURRENT & -7 & 3 & 0 & 10 & 0 & 10 & clear & mostly clear \\
\hline 85. TISDALE & -10 & -4 & 10 & 15 & 30 & 35 & clear & clear \\
\hline 86. TOMPKINS & 0 & +8 & 10 & 20 & 0 & 0 & & partly cloudy \\
\hline 87. VAL MARIE & -5 & -1 & 0 & 14 & 0 & 4 & mostly clear & clear \\
\hline 88. VANSCOY-DELISLE & -15 & -10 & 0 & 10 & 0 & 20 & & \\
\hline 89. WARMAN & -12 & -8 & 0 & 10 & 30 & 70 & mostly clear & clear \\
\hline 90. WAUCHOPE & -10 & -4 & 0 & 15 & 25 & 50 & partly cloudy & mostly cloudy, heavy fog \\
\hline 91. WEYBURN & -3 & 0 & 30 & 35 & 8 & 12 & mostly clear & mostly clear \\
\hline 92. WHITE BEAR & -12 & -5 & 0 & 1 & 0 & 15 & clear & clear \\
\hline 93. WHITE BEAR LAKE & -7 & -2 & 0 & 12 & 9 & 18 & overcast & overcast \\
\hline 94. WHITEBEECH & -3 & -4 & 0 & 0 & 28 & 30 & & overcast \\
\hline 95. WHITEWOOD & -10 & -4 & 0 & 20 & 30 & 50 & clear & clear \\
\hline 96. YORKTON & -5 & 0 & 0 & 5 & & 60 & overcast, heavy fog & overcast, heavy fog \\
\hline 97. CARRAGANA & & & & & 45 & 90 & & \\
\hline 98. CHRISTOPHER LAKE & & -2 & 0 & 0 & 45 & 60 & clear & \\
\hline 99. TURTLE LAKE & -15 & -8 & 0 & 0 & & & mostly clear & mostly clear \\
\hline
\end{tabular}

88. VANSCOY-DELISLE. Linda Lahey, A.R. Smith, Lois Smith, J.R. Smith. (4)

89. WARMAN. Terry Armstrong, Todd Arnold, Pam Martin, Lauraine Newell. (4)

90. WAUCHOPE. Dale Hjertaas, Warren Hjertaas. (2)

91. WEYBURN. Leo Belanger, L. Belanger, Ray Belanger, G. Bobbitt, E. Brodie, J. Burge, R. Churchill, R. Douglas, F. Garner, R. Gutfriend, B. Layh, P. Layh, C. Marcotte, R. Neville, N. Postey, J. Richelhof, H. Stairmand, S. Stairmand, G. Vince. (19)

92. WHITE BEAR. Darryl Jordheim, Floyd Jordheim, Gary Jordheim, Sig Jordheim. (4)

93. WHITE BEAR LAKE. Leo Belanger, Ray Belanger, Greg Bobbitt, Ross Douglas, Dick Gutfriend, Nick Postey. (6)

94. WHITEBEECH. John Kereliuk, Ed Terleski, Ida Wotherspoon, Lindsay Wotherspoon. (4)

95. WHITEWOOD. Pat Connelly, Boyd Metzler, John Pollock. (3)

96. YORKTON. Hazel Bjorgen, Warren Hjertaas, James Rushowick, Jeff Rushowick, Patrick Rushowick, Darlene Stakiw, Morris Stakiw, Dorothy Skene, Ed Skene, Harold Wilkinson. (10)
97. CARRAGANA. Stan Back, Agnes Chapman, David Chapman, Bill and Eleanor Howse, May Mueller, Margaret Sokoluk. (7)

98. CHRISTOPHER LAKE. Blake and Stella Jones, Cliff Matthews. (3)

99. TURTLE LAKE. E.M. Robinson, Marie Robinson. (2)

\section{Counts Received Too Late To Include In Table 3.}

Although these counts arrived too late to include in their proper place in Table 3 the numbers have been incorporated into the three summary columns at the end of Table 3. All other information relating to these counts are in the appropriate tables except that they are not in the proper alphabetical order. The counts and their reference numbers and the species which should be in Table 3 are reported below in text form:

\section{CARRAGANA. 26 December 1991}

Gray Partridge, 6; Ruffed Grouse, 3; Northern Hawk Owl, +; Downy Woodpecker, 2; Hairy Woodpecker, 2; Pileated Woodpecker, 1; Gray Jay, 2; Blue Jay, 4; Black-billed Magpie, 4; Common Raven, 3; Black-capped Chickadee, 5; White-breasted Nuthatch, 2; Bohemian Waxwing, 6; Snow Bunting, 55; Pine Grosbeak, 18; Common Redpoll, 15; Evening Gros- 


\section{COUNT NAME}

\begin{tabular}{|c|c|c|c|c|c|c|c|c|c|c|c|c|c|c|c|c|c|}
\hline COUNT NAME & 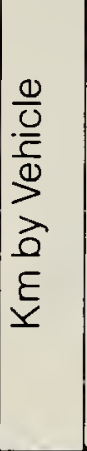 & 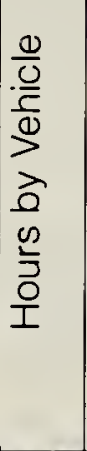 & 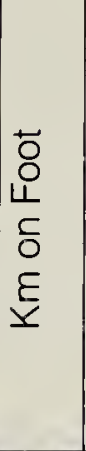 & 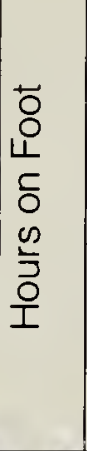 & 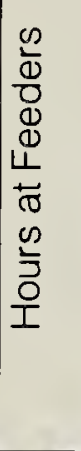 & $\begin{array}{l}0 \\
0 \\
0 \\
0 \\
3 \\
0 \\
0 \\
0 \\
\frac{0}{0} \\
\frac{0}{5} \\
0 \\
0\end{array}$ & 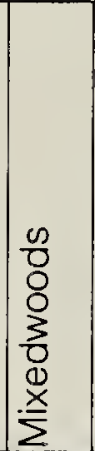 & $\begin{array}{l}0 \\
0 \\
0 \\
0 \\
3 \\
0 \\
0 \\
0 \\
0 \\
\frac{0}{0} \\
\frac{0}{0} \\
0 \\
0\end{array}$ & 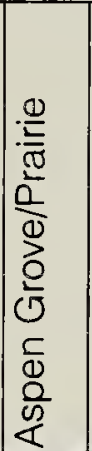 & 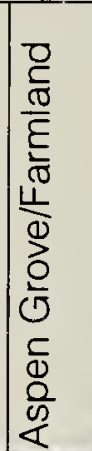 & 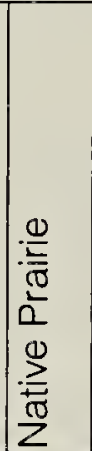 & $\begin{array}{l}0 \\
0 \\
2 \\
0 \\
0 \\
0 \\
0 \\
0 \\
0 \\
0 \\
\Phi \\
\mathbb{D} \\
0\end{array}$ & 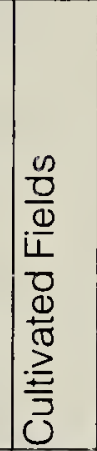 & $\begin{array}{l}0 \\
\frac{0}{2} \\
\frac{2}{2} \\
\text { 인 }\end{array}$ & $\sum_{\substack{\vdots \\
0}}^{5}$ & $\begin{array}{l}\frac{1}{0} \\
\frac{1}{\pi} \\
3 \\
\frac{1}{1} \\
0 \\
0\end{array}$ & 㐫 \\
\hline $\begin{array}{l}\text { 1. ABERNETHY-KATEPWAL. } \\
\text { 2. ARMIT* } \\
\text { 3. BANGOR } \\
\text { 4. BATTLEFORD } \\
\text { 5. BETHUNE }\end{array}$ & $\begin{array}{r}145 \\
203 \\
75 \\
25\end{array}$ & \begin{tabular}{r|}
6 \\
10 \\
2 \\
2.5
\end{tabular} & $\begin{array}{r}1 \\
16 \\
7\end{array}$ & $\begin{array}{r}.5 \\
7 \\
3 \\
.75 \\
\end{array}$ & 2 & $\begin{array}{r}6-25 \\
<5\end{array}$ & $26-50$ & $\mid \begin{array}{r}6-25 \\
26-50\end{array}$ & & $51-75$ & $<5$ & & $\begin{array}{r}6-25 \\
26-50\end{array}$ & $\begin{array}{r}<5 \\
<5 \\
26-50\end{array}$ & $\begin{array}{r}6-25 \\
<5 \\
\\
>75\end{array}$ & $<5$ & \\
\hline $\begin{array}{l}\text { 6. BIGGAR* } \\
\text { 7. BIG MUDDY LAKE* } \\
\text { 8. BIG RIVER } \\
\text { 9. BIRCH HILLS } \\
\text { 10. BORDEN }\end{array}$ & $\begin{array}{r}172 \\
101 \\
3 \\
142 \\
75 \\
\end{array}$ & $\begin{array}{r}11.5 \\
8 \\
.25 \\
5 \\
3 \\
\end{array}$ & \begin{tabular}{l|}
2 \\
1 \\
1
\end{tabular} & $\begin{array}{r}75 \\
1.5 \\
1\end{array}$ & $\begin{array}{r}1.5 \\
2\end{array}$ & $26-50$ & & $\begin{array}{l}6-25 \\
6-25\end{array}$ & & $\begin{array}{r}6-25 \\
51-75\end{array}$ & & & $51-75$ & $26-50$ & $\begin{array}{r}6-25 \\
26-50 \\
6-25\end{array}$ & & \\
\hline $\begin{array}{l}\text { 11. BRIGHTWATER RESERVOIR } \\
\text { 12. BROADVIEW } \\
\text { 13. BROMHEAD* } \\
\text { 14. CANDLE LAKE } \\
\text { 15. CHOICELAND-GRONLID }\end{array}$ & $\begin{array}{l}105 \\
103 \\
166 \\
167 \\
130 \\
\end{array}$ & \begin{tabular}{r|}
3 \\
3.5 \\
6.5 \\
8 \\
4 \\
\end{tabular} & \begin{tabular}{l|}
5 \\
5 \\
1 \\
2 \\
2 \\
\end{tabular} & \begin{tabular}{r|}
5 \\
3 \\
.5 \\
1.5 \\
2 \\
\end{tabular} & 1 & \begin{tabular}{|l|}
$26-50$ \\
$26-50$ \\
\end{tabular} & $\begin{array}{l}26-50 \\
26-50 \\
\end{array}$ & $26-50$ & $\begin{array}{r}6-25 \\
51-75 \\
\end{array}$ & $26-50$ & $6-25$ & $<5$ & $\begin{array}{l}6 \cdot 25 \\
>75\end{array}$ & $\begin{array}{r}6-25 \\
<5\end{array}$ & $6-25$ & $6-25$ & \\
\hline $\begin{array}{l}\text { 16. CLARK'S CROSSING } \\
\text { 17. CONQUEST-OUTLOOK } \\
\text { 18. CRAVEN } \\
\text { 19. CROOKED LAKE(A) } \\
\text { 20. CROOKED LAKE(B) }\end{array}$ & $\begin{array}{r}368 \\
105 \\
200 \\
140 \\
30\end{array}$ & $\begin{array}{r}14.5 \\
3 \\
12.5 \\
5 \\
2 \\
\end{array}$ & \begin{tabular}{r|}
6.5 \\
3 \\
6 \\
5 \\
2
\end{tabular} & $\begin{array}{r}5.25 \\
1 \\
5.75 \\
2 \\
25 \\
75\end{array}$ & 2 & & & $\begin{array}{r}6-25 \\
<5 \\
26-50 \\
>75\end{array}$ & $\begin{array}{r}<5 \\
<5 \\
6-25 \\
6-25\end{array}$ & $\begin{array}{r}26-50 \\
<5 \\
6-25\end{array}$ & $\begin{array}{l}<5 \\
<5\end{array}$ & $<5$ & $\mid \begin{array}{c}26-50 \\
26-50 \\
6-25\end{array}$ & $6-25$ & $\begin{array}{r}6-25 \\
6-25 \\
<5\end{array}$ & $\begin{array}{l}<5 \\
<5\end{array}$ & $<5$ \\
\hline $\begin{array}{l}\text { 21. CROOKED RIVER } \\
\text { 22. DALMENY } \\
\text { 23. DILKE } \\
\text { 24. DUVAL } \\
\text { 25. EDAM }\end{array}$ & $\begin{array}{r}100 \\
120 \\
142 \\
40 \\
\end{array}$ & $\begin{array}{r}3.25 \\
5.5 \\
2 \\
\end{array}$ & \begin{tabular}{r|}
1 \\
19 \\
7 \\
4
\end{tabular} & $\begin{array}{r}1 \\
5 \\
3.5 \\
1.5\end{array}$ & $\begin{array}{r}5 \\
10 \\
\end{array}$ & $6-25$ & $6-25$ & $6-25$ & $\begin{array}{c}6-25 \\
51-75\end{array}$ & $\begin{array}{r}26-50 \\
6-25 \\
26-50\end{array}$ & $\begin{array}{r}<5 \\
6-25\end{array}$ & $<5$ & $\begin{array}{r}26-50 \\
51-75 \\
6-25 \\
26-50\end{array}$ & \begin{tabular}{|r}
$6-25$ \\
$6-25$ \\
$<5$ \\
$6-25$
\end{tabular} & $\begin{array}{r}6-25 \\
<5 \\
<5 \\
6-25\end{array}$ & $<5$ & \\
\hline $\begin{array}{l}\text { 26. ENDEAVOUR } \\
\text { 27. FENTON } \\
\text { 28. FORT QU'APPELLE } \\
\text { 29. FORT WALSH* } \\
\text { 30. GARDINER DAM* }\end{array}$ & $\begin{array}{r}40 \\
152 \\
100 \\
174 \\
287 \\
\end{array}$ & \begin{tabular}{r|}
3.5 \\
4 \\
8 \\
15 \\
5.5 \\
\end{tabular} & $\begin{array}{r}2 \\
38 \\
13 \\
\end{array}$ & $\begin{array}{r}1 \\
16.5 \\
6.5 \\
\end{array}$ & $\begin{array}{l}1 \\
8 \\
1\end{array}$ & & $51-75$ & $6-25$ & & $\begin{array}{r}51-75 \\
6-25 \\
6-25 \\
\\
<5 \\
\end{array}$ & $<5$ & & $\mid \begin{array}{r}51-75 \\
6-25 \\
6-25 \\
26-50\end{array}$ & $\begin{array}{r}6-25 \\
6-25 \\
6-25 \\
26-50 \\
\end{array}$ & $6-25$ & $\begin{array}{r}<5 \\
26-50 \\
\end{array}$ & $\begin{array}{l}6-25 \\
6-25 \\
\end{array}$ \\
\hline $\begin{array}{l}\text { 31. GOOD SPIRIT LAKE* } \\
\text { 32. GLENTWORTH } \\
\text { 33. GOVENLOCK* } \\
\text { 34. GRASSLANDS NAT. PARK } \\
\text { 35. GRAYSON }\end{array}$ & $\begin{array}{r}100 \\
182 \\
316 \\
211 \\
50 \\
\end{array}$ & \begin{tabular}{r|}
4 \\
3 \\
13 \\
5 \\
5 \\
\end{tabular} & $\begin{array}{r}5 \\
9 \\
16 \\
3 \\
8\end{array}$ & \begin{tabular}{r|}
2.5 \\
5 \\
8 \\
2 \\
1 \\
\end{tabular} & 1 & $<5$ & $<5$ & $6-25$ & $\begin{array}{r}6-25 \\
<5\end{array}$ & $\begin{array}{l}26-50 \\
26-50 \\
\end{array}$ & $\begin{array}{r}<5 \\
>75 \\
51-75 \\
51-75 \\
\end{array}$ & $6-25$ & $\begin{array}{r}26-50 \\
<5 \\
26-50 \\
6-25 \\
6-25\end{array}$ & $\begin{array}{r}6-25 \\
<5 \\
6-25 \\
6-25 \\
6-25 \\
\end{array}$ & $<5$ & $<5$ & $6-25$ \\
\hline $\begin{array}{l}\text { 36. GRENFELL } \\
\text { 37. HEPBURN } \\
\text { 38. HUDSON BAY* } \\
\text { 39. HUMBOLDT } \\
\text { 40. INDIAN HEAD }\end{array}$ & $\begin{array}{r}348 \\
50 \\
200 \\
\end{array}$ & $\begin{array}{r}12 \\
2 \\
8 \\
\end{array}$ & $\begin{array}{r}12 \\
1 \\
10 \\
\end{array}$ & $\begin{array}{l}5 \\
1 \\
7 \\
\end{array}$ & $\begin{array}{l}3 \\
1 \\
6\end{array}$ & $6-25$ & $6-25$ & $6-25$ & $5-25$ & \begin{tabular}{|l|}
$26-50$ \\
$26-50$ \\
\end{tabular} & $\begin{array}{r}<5 \\
6-25 \\
\end{array}$ & & $\begin{array}{r}6-25 \\
26-50 \\
26-50 \\
\end{array}$ & $\begin{array}{c}6-25 \\
6-25\end{array}$ & $\begin{array}{r}26-50 \\
6-25 \\
6-25\end{array}$ & $\begin{array}{l}<5 \\
<5 \\
\end{array}$ & \\
\hline $\begin{array}{l}\text { 41. KAMSACK } \\
\text { 42. KELVINGTON } \\
\text { 43. KENASTON } \\
\text { 44. KILWINNING } \\
\text { 45. KINDERSLEY }\end{array}$ & $\begin{array}{r}40 \\
90 \\
67 \\
164\end{array}$ & $\begin{array}{r}2 \\
9 \\
4.5 \\
4.5 \\
\end{array}$ & 1 & 1 & $\begin{array}{r}18 \\
5\end{array}$ & $26-50$ & $6-25$ & $6-25$ & $6-25$ & $\begin{array}{r}6-25 \\
6-25 \\
51-75 \\
26-50 \\
51-75 \\
\end{array}$ & $\begin{array}{l}<5 \\
<5\end{array}$ & & $\begin{array}{r}6-25 \\
26-50 \\
6-25 \\
\end{array}$ & $\begin{array}{r}51-75 \\
<5\end{array}$ & $\begin{array}{r}6-25 \\
<5 \\
<5 \\
\end{array}$ & & $<5$ \\
\hline $\begin{array}{l}\text { 46. KINLOCH } \\
\text { 47. KUTAWAGAN LAKE } \\
\text { 48. LARONGE } \\
\text { 49. LAST MOUNTAIN L. W.M.U.* } \\
\text { 50. LEADER }\end{array}$ & $\begin{array}{r}50 \\
201 \\
104 \\
260 \\
50 \\
\end{array}$ & $\begin{array}{r}2.5 \\
4 \\
5.5 \\
6 \\
1 \\
\end{array}$ & \begin{tabular}{l|} 
\\
1 \\
2 \\
2 \\
6 \\
\end{tabular} & $\begin{array}{r}.5 \\
1 \\
.5 \\
2 \\
3 \\
\end{array}$ & 8 & $\begin{array}{r}<5 \\
6-25\end{array}$ & $\begin{array}{c}6-25 \\
51-75\end{array}$ & $6-25$ & & $26-50$ & $\begin{array}{r}6-25 \\
\\
6-25 \\
26-50 \\
\end{array}$ & $6-25$ & $\begin{array}{r}>75 \\
>75 \\
6-25 \\
\end{array}$ & \begin{tabular}{|r|}
$<5$ \\
$6-25$ \\
$6-25$ \\
\end{tabular} & $6-25$ & $<5$ & \\
\hline $\begin{array}{l}\text { 51. LIVELONG } \\
\text { 52. LOVE-TORCH RIVER } \\
\text { 53. LUSELAND } \\
\text { 54. MACDOWALL } \\
\text { 55. MATADOR }\end{array}$ & $\begin{array}{r}138 \\
72 \\
80 \\
98\end{array}$ & $\begin{array}{r}5.5 \\
2.5 \\
3.25 \\
5\end{array}$ & $\begin{array}{r}4 \\
1 \\
16.5 \\
11 \\
5\end{array}$ & \begin{tabular}{r|}
1 \\
1 \\
12 \\
3.25 \\
2
\end{tabular} & $\begin{array}{r}4 \\
10 \\
1 \\
12.5\end{array}$ & $<5$ & $\left|\begin{array}{r}26-50 \\
6-25\end{array}\right|$ & & $\begin{array}{r}6-25 \\
51-75\end{array}$ & \begin{tabular}{|r|}
$6-25$ \\
$26-50$ \\
$26-50$ \\
\end{tabular} & $\begin{array}{r}<5 \\
51-75\end{array}$ & $\begin{array}{c}6-25 \\
6-25\end{array}$ & $\begin{array}{c}6-25 \\
6-25\end{array}$ & $\begin{array}{r}6-25 \\
6-25 \\
<5 \\
\end{array}$ & $\begin{array}{r}>75 \\
<5 \\
6-25\end{array}$ & & $26-50$ \\
\hline $\begin{array}{l}\text { 56. MEADOW LAKE } \\
\text { 57. MELFORT } \\
\text { 58. MELVILLE } \\
\text { 59. MOOSE JAW } \\
\text { 60. NAICAM }\end{array}$ & $\begin{array}{r}100 \\
20 \\
40 \\
113 \\
111\end{array}$ & $\begin{array}{r}3.5 \\
3 \\
1 \\
7 \\
5 \\
\end{array}$ & $\begin{array}{r}4 \\
4 \\
1 \\
14 \\
1 \\
\end{array}$ & $\begin{array}{r}3 \\
2 \\
5 \\
11 \\
1 \\
\end{array}$ & $\begin{array}{r}10 \\
1 \\
2 \\
2 \\
4\end{array}$ & $\begin{array}{r}6-25 \\
<5\end{array}$ & $6-25$ & $6-25$ & & $\begin{array}{r}6-25 \\
26-50 \\
26-50 \\
\end{array}$ & & & $\begin{array}{r}51-75 \\
6-25 \\
6-25 \\
26-50\end{array}$ & $\begin{array}{r}<5 \\
51-75 \\
\end{array}$ & $\begin{array}{l}6-25 \\
6-25 \\
6-25\end{array}$ & & $26-50$ \\
\hline $\begin{array}{l}\text { 61. NEELY LAKE } \\
\text { 62. NIPAWIN } \\
\text { 63. PIKE LAKE } \\
\text { 64. PORCUPINE PLAIN } \\
\text { 65. PREECEVILLE }\end{array}$ & $\begin{array}{r}52 \\
6 \\
342 \\
100 \\
50\end{array}$ & $\begin{array}{r}4 \\
.25 \\
8.25 \\
4 \\
2.25\end{array}$ & \begin{tabular}{r|}
1 \\
3 \\
27 \\
2 \\
3.5
\end{tabular} & $\begin{array}{r}1 \\
2 \\
23.5 \\
2 \\
2.75\end{array}$ & $\begin{array}{r}7 \\
2 \\
16 \\
4\end{array}$ & $<5$ & $\begin{array}{l}6-25 \\
>75 \\
6-25 \\
6-25\end{array}$ & \begin{tabular}{|r|}
$26-50$ \\
$26-50$ \\
$6-25$ \\
$6-25$
\end{tabular} \mid & $<5$ & $\begin{array}{c}6-25 \\
6-25\end{array}$ & & $\begin{array}{l}<5 \\
<5\end{array}$ & $\begin{array}{r}6-25 \\
6-25 \\
26-50 \\
6-25\end{array}$ & $\begin{array}{c}6-25 \\
6-25 \\
6-25 \\
6-25\end{array}$ & $\begin{array}{r}6-25 \\
26-50 \\
6-25\end{array}$ & $<5$ & $<5$ \\
\hline
\end{tabular}

\begin{tabular}{|c|c|c|c|c|c|c|c|c|c|c|c|c|c|c|c|c|c|}
\hline OUNT NAME & $\begin{array}{l}\frac{0}{0} \\
\frac{0}{2} \\
\frac{0}{2} \\
\frac{\partial}{2} \\
\frac{\partial}{x} \\
\end{array}$ & 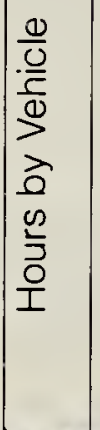 & 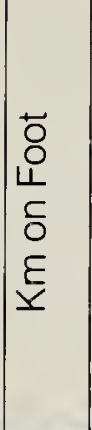 & 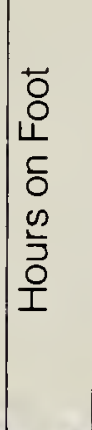 & 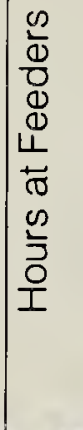 & $\begin{array}{l}0 \\
0 \\
0 \\
0 \\
0 \\
0 \\
0 \\
0 \\
0.0 \\
0 \\
0 \\
0\end{array}$ & $\begin{array}{l}0 \\
0 \\
8 \\
0 \\
3 \\
0 \\
0 \\
x \\
\\
\Sigma\end{array}$ & $\begin{array}{l}0 \\
0 \\
0 \\
3 \\
0 \\
0 \\
0 \\
0 \\
\frac{0}{0} \\
0 \\
0\end{array}$ & 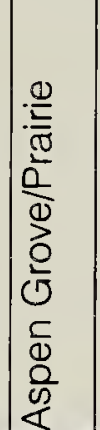 & 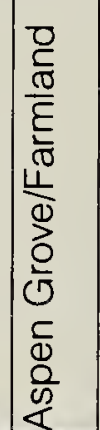 & 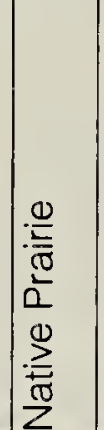 & 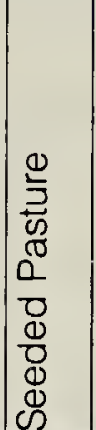 & $\begin{array}{l}\frac{0}{0} \\
\frac{0}{1} \\
\frac{0}{0} \\
\frac{0}{0} \\
\frac{0}{3} \\
\frac{3}{3} \\
0\end{array}$ & 足 & $\underbrace{\substack{0 \\
0}}_{0}$ & 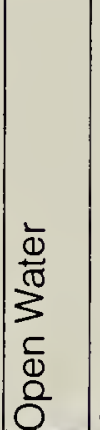 & 产 \\
\hline $\begin{array}{l}\text { 1. ABERNETHY-KATEPWAL } \\
\text { 2. ARMIT* } \\
\text { 3. BANGOR } \\
\text { 4. BATLEFORD } \\
\text { 5. BETHUNE }\end{array}$ & \begin{tabular}{r|}
145 \\
203 \\
75 \\
25
\end{tabular} & $\begin{array}{r}6 \\
10 \\
2\end{array}$ & $\begin{array}{r}1 \\
16 \\
7\end{array}$ & $\begin{array}{l}5 \\
7 \\
3\end{array}$ & 2 & $\begin{array}{r}6-25 \\
<5\end{array}$ & $26-50$ & $\mid \begin{array}{r}6-25 \\
26-50\end{array}$ & & $51-75$ & $<5$ & & $\left|\begin{array}{r}6-25 \\
26-50\end{array}\right|$ & $\begin{array}{r}<5 \\
<5 \\
26-50\end{array}$ & $\begin{array}{r}6-25 \\
<5 \\
>75 \\
\end{array}$ & $<5$ & \\
\hline $\begin{array}{l}\text { 6. BIGGAR* } \\
\text { 7. BIG MUDDY LAKE* } \\
\text { 8. BIG RIVER } \\
\text { 9. BIRCH HILLS } \\
\text { 10. BORDEN } \\
\end{array}$ & $\begin{array}{r}172 \\
101 \\
3 \\
142 \\
75 \\
\end{array}$ & $\begin{array}{r}11.5 \\
8 \\
25 \\
5 \\
3 \\
\end{array}$ & \begin{tabular}{l|l}
2 \\
1 \\
1
\end{tabular} & $\begin{array}{r}75 \\
1.5 \\
1 \\
\end{array}$ & $\begin{array}{r}1.5 \\
2\end{array}$ & $26-50$ & & \begin{tabular}{|r|}
$6-25$ \\
$6-25$ \\
\end{tabular} & & $\begin{array}{r}6-25 \\
51-75 \\
\end{array}$ & & & $51-75$ & $26-50$ & $\left|\begin{array}{r}26-50 \\
6-25\end{array}\right|$ & & \\
\hline $\begin{array}{l}\text { ATERRESERVOR } \\
\text { IEW } \\
\text { AD* } \\
\text { LAKE } \\
\text { AND-GRONLID }\end{array}$ & $\begin{array}{l}105 \\
103 \\
166 \\
167 \\
130\end{array}$ & \begin{tabular}{r|}
3 \\
3.5 \\
6.5 \\
8 \\
4 \\
\end{tabular} & $\begin{array}{l}5 \\
5 \\
1 \\
2 \\
\end{array}$ & $\begin{array}{r}5 \\
3 \\
.5 \\
1.5\end{array}$ & 1 & $\left|\begin{array}{l}26-50 \\
26-50\end{array}\right|$ & $\mid \begin{array}{l}26-50 \\
26-50\end{array}$ & $51-75$ & \begin{tabular}{|r|}
$6-25$ \\
$51-75$ \\
\end{tabular} & 26-50 & $6-25$ & $<5$ & 6.25 & $\begin{array}{r}6-25 \\
<5\end{array}$ & $6-25$ & $6-25$ & \\
\hline $\begin{array}{l}\text { 16. CLARK'S CROSSING } \\
\text { 17. CONQUEST-OUTLOOK } \\
\text { 18. CRAVEN } \\
\text { 19. CROOKED LAKE(A) } \\
\text { 20. CROOKED LAKE(B) }\end{array}$ & $\begin{array}{r}-150 \\
105 \\
200 \\
140 \\
30\end{array}$ & \begin{tabular}{|r|}
14.5 \\
3 \\
12.5 \\
5 \\
2 \\
\end{tabular} & \begin{tabular}{r|}
6.5 \\
3 \\
6 \\
5 \\
2
\end{tabular} & \begin{tabular}{r|}
5.25 \\
1 \\
5.75 \\
2 \\
.75
\end{tabular} & 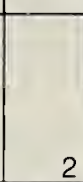 & & & \begin{tabular}{|r|}
$6-25$ \\
$<5$ \\
\end{tabular} & \begin{tabular}{|l|}
$<5$ \\
$<5$
\end{tabular} & \begin{tabular}{|r|}
$26-50$ \\
$<5$
\end{tabular} & \begin{tabular}{l|}
$<5$ \\
$<5$
\end{tabular} & $\begin{array}{l}<5 \\
<5\end{array}$ & $\mid \begin{array}{r}26-50 \\
26-50 \\
6-25\end{array}$ & $\frac{6-25}{6-25}$ & \begin{tabular}{|r|}
$6-25$ \\
$6-25$ \\
\end{tabular} & $\begin{array}{l}<5 \\
<5\end{array} \mid$ & \\
\hline $\begin{array}{l}\text { 21. CROOKED RIVER } \\
\text { 22. DALMENY } \\
\text { 23. DILKE } \\
\text { 24. DUVAL } \\
\text { 25. EDAM }\end{array}$ & $\begin{array}{r}120 \\
142 \\
40\end{array}$ & $\begin{array}{r}3.25 \\
5.5 \\
2 \\
\end{array}$ & $\begin{array}{r}1 \\
19 \\
7 \\
4\end{array}$ & $\begin{array}{r}1 \\
5 \\
3.5 \\
1.5\end{array}$ & $\begin{array}{r}5 \\
10\end{array}$ & $6-25$ & $6-25$ & $6-25$ & $\left|\begin{array}{c}6-25 \\
51-75\end{array}\right|$ & $\mid \begin{array}{r}26-50 \\
6-25 \\
26-50\end{array}$ & $\begin{array}{r}<5 \\
6-25 \\
\end{array}$ & $<5$ & $\begin{array}{r}26-50 \\
51-75 \\
6-25 \\
26-50\end{array}$ & $\mid \begin{array}{r}6-25 \\
6-25 \\
<-5 \\
6-25\end{array}$ & \begin{tabular}{|r|r|}
$6-25$ \\
$<5$ \\
$<5$ \\
$6-25$ \\
6
\end{tabular} & $<5$ & \\
\hline $\begin{array}{l}\text { AVOUR } \\
\text { ON } \\
\text { QU'APPELLE } \\
\text { WALSH* } \\
\text { DINER DAM* }\end{array}$ & \begin{tabular}{r|}
40 \\
152 \\
100 \\
174 \\
287 \\
\end{tabular} & $\begin{array}{r}3.5 \\
4 \\
8 \\
15 \\
5.5 \\
\end{array}$ & $\begin{array}{r}2 \\
38 \\
13 \\
\end{array}$ & $\begin{array}{r}1 \\
16.5 \\
6.5\end{array}$ & $\begin{array}{l}1 \\
8 \\
1\end{array}$ & & $51-75$ & $6-25$ & & \begin{tabular}{|r}
$51-75$ \\
$6-25$ \\
$6-25$ \\
\end{tabular} & $<5$ & & $\mid \begin{array}{r}51-75 \\
6-25 \\
6-25 \\
26-50\end{array}$ & \begin{tabular}{|r|}
$6-25$ \\
$6-25$ \\
$6-25$ \\
$26-50$ \\
\end{tabular} & 6-25 & $\begin{array}{r}<5 \\
26-50 \\
\end{array}$ & \\
\hline $\begin{array}{l}\text { 31. GOOD SPIRIT LAKE* } \\
\text { 32. GLENTWORTH } \\
\text { 33. GOVENLOCK } \\
\text { 34. GRASSLANDS NAT. PARK } \\
\text { 35. GRAYSON }\end{array}$ & \begin{tabular}{r|}
100 \\
182 \\
316 \\
211 \\
50
\end{tabular} & \begin{tabular}{r|r|}
4 \\
3 \\
13 \\
5 \\
5
\end{tabular} & \begin{tabular}{r|r}
5 \\
9 \\
16 \\
3 \\
8
\end{tabular} & \begin{tabular}{r|}
2.5 \\
5 \\
8 \\
2 \\
1
\end{tabular} & 2 & $<5$ & $<5$ & $6-25$ & $\begin{array}{r}6-25 \\
<5\end{array}$ & $\mid$\begin{tabular}{|c|}
$26-50$ \\
$26-50$
\end{tabular} & $\begin{array}{r}<5 \\
>75 \\
51-75 \\
51-75 \\
\end{array}$ & $6-25$ & $\mid \begin{array}{r}26-50 \\
<-5 \\
26-50 \\
6-25 \\
6-25\end{array}$ & \begin{tabular}{r|r|}
$6-25$ \\
$<-5$ \\
$6-25$ \\
$6-25$ \\
$6-25$
\end{tabular} & $<5$ & $<5$ & $6-25$ \\
\hline $\begin{array}{l}\text { 36. GRENFELL } \\
\text { 37. HEPBURN } \\
\text { 38. HUDSON BAY* } \\
\text { 39. HUMBOLDT } \\
\text { 40. INDIAN HEAD } \\
\end{array}$ & $\begin{array}{r}348 \\
50 \\
200 \\
\end{array}$ & $\begin{array}{r}12 \\
2 \\
8 \\
\end{array}$ & $\begin{array}{r}12 \\
1 \\
10 \\
\end{array}$ & \begin{tabular}{l|l}
5 \\
1 \\
7 \\
\end{tabular} & $\begin{array}{l}3 \\
1 \\
6 \\
\end{array}$ & $6-25$ & $6-25$ & $6-25 \mid$ & & $\begin{array}{r}26-50 \\
26-50 \\
\end{array}$ & $\begin{array}{r}55 \\
6-25 \\
\end{array}$ & & $\begin{array}{r}6-25 \\
26-50 \\
26-50\end{array}$ & $\left|\begin{array}{l}6-25 \\
6-25\end{array}\right|$ & $\begin{array}{r}26-50 \\
6-25 \\
6-25 \\
\end{array}$ & $<5$ & \\
\hline $\begin{array}{l}\text { 41. KAI } \\
\text { 42. KEL } \\
\text { 43. KEN } \\
\text { 44. KIL } \\
\text { 45. KIN }\end{array}$ & $\begin{array}{r}40 \\
90 \\
67 \\
164 \\
\end{array}$ & $\begin{array}{r}2 \\
9 \\
4.5 \\
4.5\end{array}$ & 1 & 1 & $\begin{array}{r}18 \\
5\end{array}$ & $26-50$ & $26-50$ & $|6-25|$ & $6-25$ & \begin{tabular}{|r|}
$6-25$ \\
$6-25$ \\
$51-75$ \\
$26-50$ \\
$51-75$ \\
\end{tabular} & $\begin{array}{l}<5 \\
<5 \\
\end{array}$ & & \begin{tabular}{|c|}
$6-25$ \\
$26-50$ \\
$6-25$ \\
\end{tabular} & $<5$ & \begin{tabular}{|r|}
$6-25$ \\
$<5$ \\
$<5$ \\
\end{tabular} & & e \\
\hline $\begin{array}{l}\text { GAN LAKE } \\
\text { INTAIN L. W.M.U." }\end{array}$ & $\begin{array}{r}50 \\
201 \\
104 \\
260 \\
50 \\
\end{array}$ & $\begin{array}{r}2.5 \\
4 \\
5.5 \\
6\end{array}$ & $\begin{array}{l}1 \\
1 \\
2 \\
2\end{array}$ & \begin{tabular}{r|}
.5 \\
1 \\
.5 \\
2 \\
3 \\
\end{tabular} & 8 & $\begin{array}{r}<5 \\
6-25\end{array}$ & \begin{tabular}{|c|}
$6-25$ \\
$51-75$
\end{tabular} & $6-25$ & & $26-50$ & $\begin{array}{r}6-25 \\
26-50\end{array}$ & $6-25$ & \begin{tabular}{|c|}
$>75$ \\
$6-25$ \\
\end{tabular} & $\begin{array}{r}6-25 \\
6-25 \\
\end{array}$ & $6-25$ & $<5$ & \\
\hline $\begin{array}{l}\text { H RIVER } \\
\text { L }\end{array}$ & $\begin{array}{r}138 \\
72 \\
80 \\
98\end{array}$ & $\begin{array}{r}5.5 \\
2.5 \\
3.25 \\
5\end{array}$ & $\begin{array}{r}4 \\
1 \\
16.5 \\
11 \\
5\end{array}$ & $\begin{array}{r}1 \\
1 \\
12 \\
3.25 \\
2\end{array}$ & $\begin{array}{r}4 \\
10 \\
1 \\
12.5\end{array}$ & $<5$ & $26-50$ & & $\begin{array}{r}6-25 \\
51-75\end{array}$ & \begin{tabular}{|r|}
$6-25$ \\
$26-50$ \\
$26-50$ \\
\end{tabular} & $<5$ & $6-25$ & $6-25$ & $\begin{array}{r}6-25 \\
6-25 \\
<5 \\
\end{array}$ & $\begin{array}{r}>75 \\
<5 \\
6-25\end{array}$ & & \\
\hline $\begin{array}{l}\text { 56. MEADOW LAKE } \\
\text { 5. MELFORT } \\
\text { 5. MELVILLE } \\
\text { 59. MOOSE JAW } \\
\text { 50. NAICAM }\end{array}$ & \begin{tabular}{r|r}
100 \\
20 \\
40 \\
113 \\
111
\end{tabular} & $\begin{array}{r}3.5 \\
3 \\
1 \\
7 \\
5\end{array}$ & $\begin{array}{r}4 \\
4 \\
1 \\
14 \\
1\end{array}$ & $\begin{array}{r}3 \\
2 \\
.5 \\
11\end{array}$ & $\begin{array}{r}10 \\
1 \\
2\end{array}$ & $\begin{array}{r}6-25 \\
<5\end{array}$ & $6-25$ & 6-25 & & 26-50 & & & r $\begin{array}{r}51-75 \\
6-25 \\
6-25 \\
26-50\end{array}$ & $\begin{array}{r}<5 \\
51-75 \\
\end{array}$ & \begin{tabular}{|l|}
$6-25$ \\
$6-25$ \\
$6-25$
\end{tabular} & & \\
\hline $\begin{array}{l}\text { 51. NEELLY LAKE } \\
\text { 22. NIPAWIN } \\
\text { 33. PIKE LAKE } \\
\text { 54. PORCUPINE PLAIN } \\
\text { 55. PREECEVILLE }\end{array}$ & \begin{tabular}{r|}
52 \\
6 \\
342 \\
100 \\
50
\end{tabular} & \begin{tabular}{r|} 
\\
.25 \\
8.25 \\
4 \\
2.25 \\
\end{tabular} & $\begin{array}{r}3 \\
27 \\
2 \\
3.5\end{array}$ & $\begin{array}{r}23.5 \\
2 \\
2.75 \\
\end{array}$ & $\begin{array}{r}16 \\
4\end{array}$ & & $\begin{array}{l}6-25 \\
6-25 \\
\end{array}$ & \begin{tabular}{|r|}
$26-50$ \\
$26-50$ \\
$6-25$ \\
$6-25$ \\
\end{tabular} & $<5$ & $\begin{array}{l}6-25 \\
6-25\end{array}$ & & $\begin{array}{l}<5 \\
<5\end{array}$ & $\mid \begin{array}{r}6-25 \\
26-50 \\
6-25\end{array}$ & $\begin{array}{l}6-25 \\
6-25 \\
6-25\end{array} \mid$ & $\begin{array}{r}6-25 \\
26-50 \\
6-25 \\
\end{array}$ & $<5$ & $<5$ \\
\hline
\end{tabular}

\section{Habitat Covered (\%)}

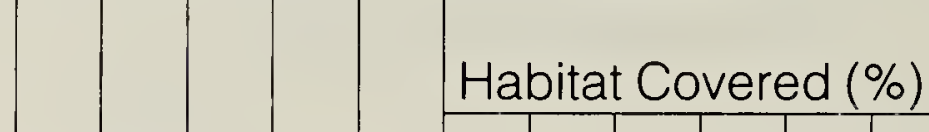


Table 2. COUNT COVERAGE (Continued) ( ${ }^{*}$ indicates counts which conform to the North American standard of all day counts covering a 15 mile diameter circle)

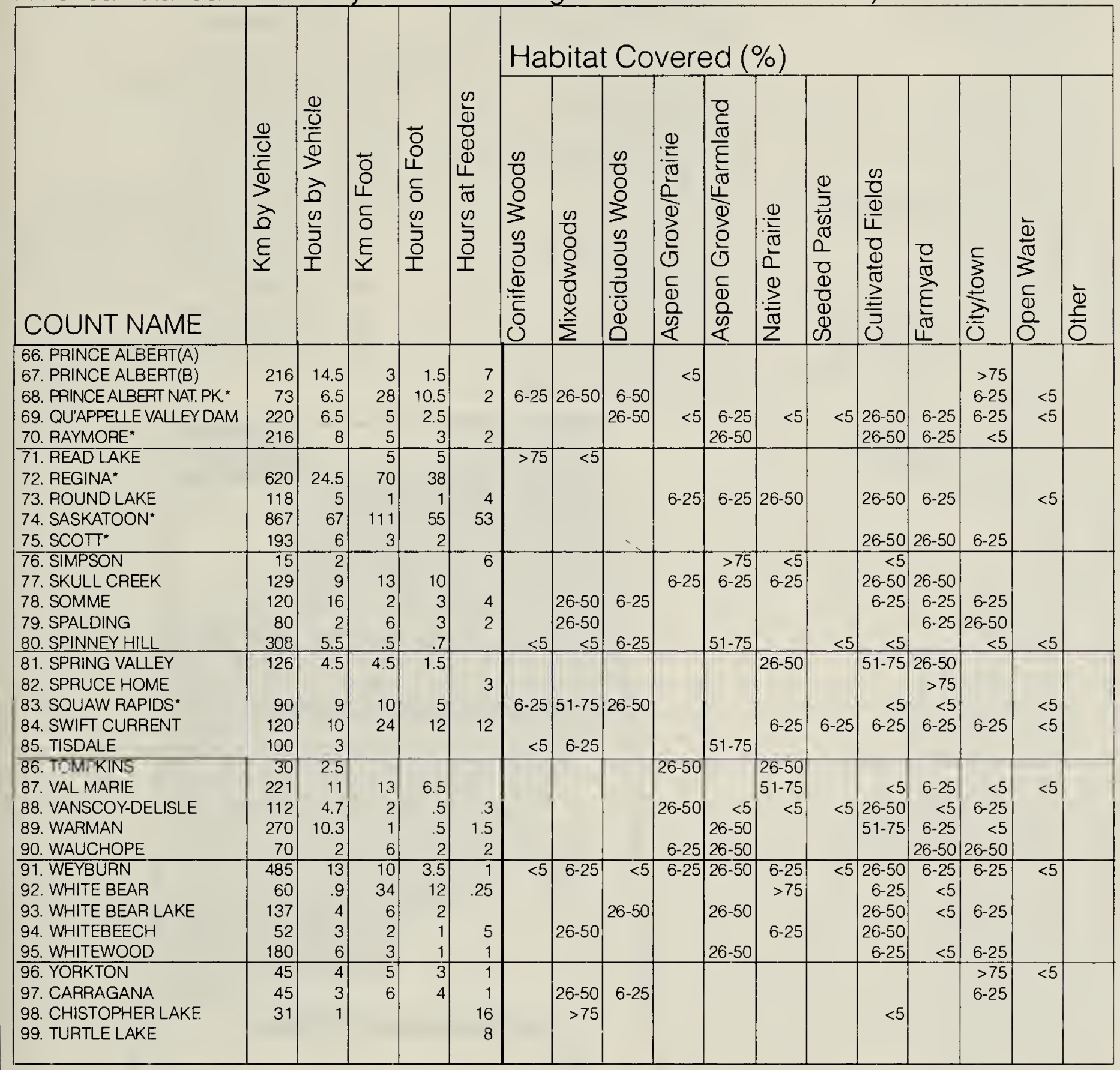

beak, 20; House Sparrow, 4. Total species, 16; Total count period species, 17; Species in Table 4 \& 5, 0; Individuals in Table $4 \& 5,0$; Total count day individuals, 152 .

98. CHRISTOPHER LAKE. 26 December 1991. Ruffed Grouse, 2; Northern Hawk Owl, 1; Downy Woodpecker, 1; Hairy Woodpecker, 1; Blue Jay, 4; Black-billed Magpie, 1; Common Raven, 1; Black-capped Chickadee, 4; Red-breasted Nuthatch, 1; Whitebreasted Nuthatch, 1; Common Redpoll, 2; Evening Grosbeak, 1. Total species, 12; Total count period species, 12; Species in Table 4 \& 5, 0;
Individuals in Table 4 \& 5, 0; Total count day individuals, 20 .

99. TURTLE LAKE. 2 January 1992. Bald Eagle, 1; Ruffed Grouse, 2; Great Gray Owl, 2; Downy Woodpecker, 8; Hairy Woodpecker, 10; Gray Jay, 7; Blue Jay, 10; Black-billed Magpie, 5; Common Raven, 25; Black-capped Chickadee, 15; Boreal Chickadee, 1; White-breasted Nuthatch, 4; Pine Grosbeak, 100; Common Redpoll, 25; Hoary Redpoll, 15; Evening Grosbeak, 75 . Total species, 17; Total count period species, 17 ; Species in Table 4 \& 5, 1 ; Individuals in Table 4 \& 5 , 2; Total count day individuals, 307. 
1. ABERNETHY-KATEPWA L.

2. ARMIT

3. BANGOR

4. BATTLEFORD

5. BETHUNE

6. BIGGAR

7. BIG MUDDY

8. BIG RIVER

9. BIRCH HILLS

10. BORDEN

11. BRIGHTWATER RESERVOIR

12. BROADVIEW

13. BROMHEAD

14. CANDLE LAKE

15. CHOICELAND-GRONLID

16. CLARK'S CROSSING

17. CONQUEST-OUTLOOK

18. CRAVEN

19. CROOKED LAKE (A)

20. CROOKED LAKE (B)

21. CROOKED RIVER

22. DALMENY

23. DILKE

24. DUVAL

25. EDAM

26. ENDEAVOUR

27. FENTON

28. FORT QU'APPELLE

29. FORT WALSH

30. GARDINER DAM

31. GLENTWORTH

32. GOOD SPIRIT LAKE

33. GOVENLOCK

34. GRASSLANDS NAT. PK.

35. GRAYSON DISTRICT

36. GRENFELL

37. HEPBURN

38. HUDSON BAY

39. HUMBOLDT

40. INDIAN HEAD

41. KAMSACK

42. KELVINGTON

43. KENASTON

44. KILWINNING

45. KINDERSLEY

46. KINLOCH

47. KUTAWAGAN LAKE

48. LA RONGE

49. LAST MOUNTAIN LAKE

50. LEADER

51. LIVELONG

52. LOVE-TORCH RIVER

53. LUSELAND

54. MACDOWALL

55. MATADOR

56. MEADOW LAKE

57. MELFORT

58. MELVILLE

59. MOOSE JAW

60. NAICAM

61. NEELY LAKE

62. NIPAWIN

63. PIKE LAKE

64. PORCUPINE PLAIN
65. PREECEVILLE

66. PRINCE ALBERT (A)

67. PRINCE ALBERT (B)

68. PRINCE ALBERT N.P.

69. QU'APPELLE VALLEY DAM

70. RAYMORE

71. READ LAKE

72. REGINA

73. ROUND LAKE

74. SASKATOON

75. SCOTT

76. SIMPSON

77. SKULL CREEK

78. SOMME

79. SPALDING

80. SPINNEY HILL

81. SPRING VALLEY

82. SPRUCE HOME
83. SQUAW RAPIDS

84. SWIFT CURRENT

85. TISDALE

86. TOMPKINS

87. VAL MARIE

88. VANSCOY-DELISLE

89. WARMAN

90. WAUCHOPE

91. WEYBURN

92. WHITE BEAR

93. WHITE BEAR LAKE

94. WHITEBEECH

95. WHITEWOOD

96. YORKTON

97. CARRAGANA

98. CHRISTOPHER LAKE

99. TURTLE LAKE

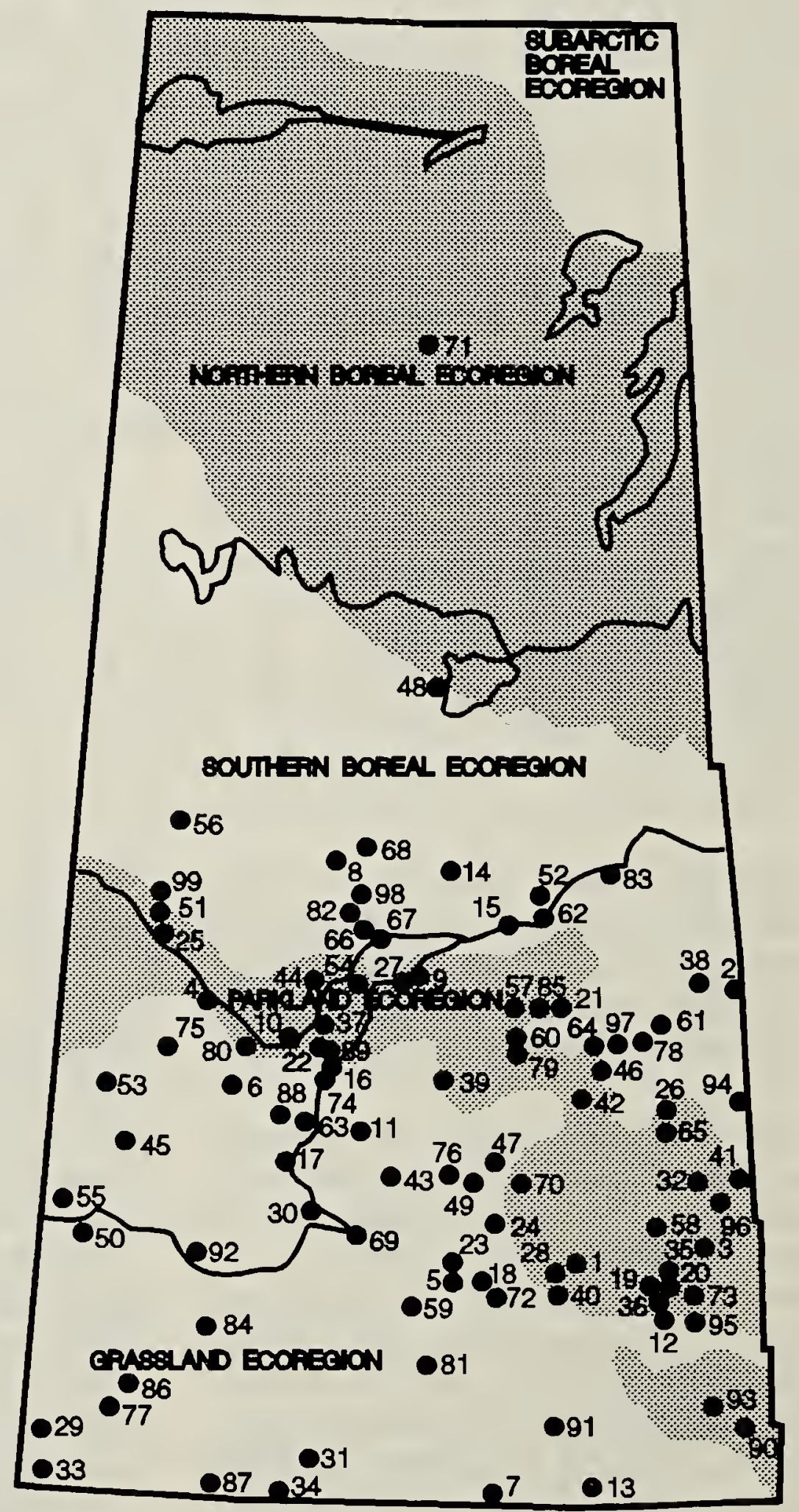

Figure 1. Location of 1991 counts. 
Table 3-1. SPECIES RECORDED FROM MORE THAN NINE LOCALITIES $(+=a$ species seen during the count period but not on count day).

\begin{tabular}{|c|c|c|c|c|c|c|c|c|c|c|}
\hline 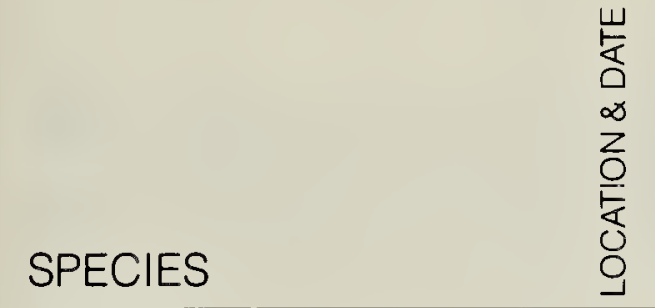 & 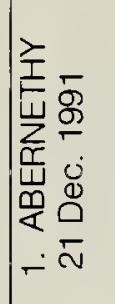 & 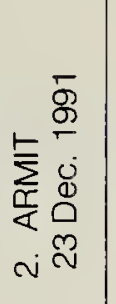 & 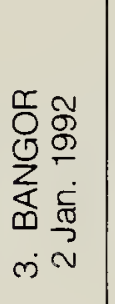 & 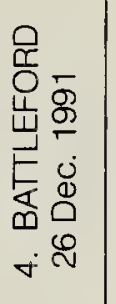 & 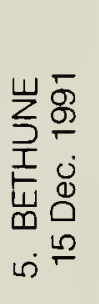 & 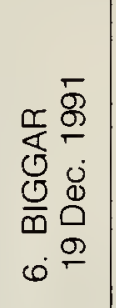 & 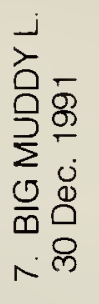 & 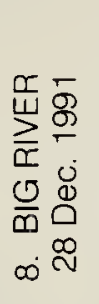 & 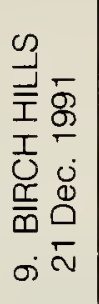 & 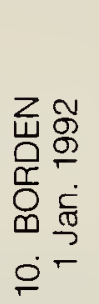 \\
\hline \multirow{5}{*}{$\begin{array}{l}\text { MALLARD } \\
\text { COMMON GOLDENEYE } \\
\text { BALD EAGLE } \\
\text { NORTHERN GOSHAWK } \\
\text { GOLDEN EAGLE }\end{array}$} & 12 & & & & & & & & & \\
\hline & 6 & & & & & & & & & 1 \\
\hline & + & & & & & & & & & 1 \\
\hline & & 1 & & & & & & & & 2 \\
\hline & 1 & 1 & + & & & 1 & 3 & & & \\
\hline \multirow{5}{*}{$\begin{array}{l}\text { MERLIN } \\
\text { PRAIRIE FALCON } \\
\text { GRAY PARTRIDGE } \\
\text { RING-NECKED PHEASANT } \\
\text { RUFFED GROUSE }\end{array}$} & + & & & & & + & & & & \\
\hline & & & & & & & 1 & & & \\
\hline & 23 & & + & & 22 & 49 & 19 & & & \\
\hline & & & & & & & 1 & & & \\
\hline & & 13 & 11 & 1 & & + & & 4 & 1 & \\
\hline \multirow{5}{*}{$\begin{array}{l}\text { SHARP-TAILED GROUSE } \\
\text { ROCK DOVE } \\
\text { GREAT HORNED OWL } \\
\text { SNOWY OWL } \\
\text { NORTHERN HAWK OWL }\end{array}$} & 34 & 1 & + & & 5 & 73 & & & & 17 \\
\hline & 6 & & & & & 174 & 30 & & 6 & 41 \\
\hline & & 2 & + & & & 2 & 2 & + & 1 & 1 \\
\hline & + & & + & & 1 & + & & & 3 & \\
\hline & & 4 & & & & & & & & 1 \\
\hline \multirow{5}{*}{$\begin{array}{l}\text { GREAT GRAY OWL } \\
\text { DOWNY WOODPECKER } \\
\text { HAIRY WOODPECKER } \\
\text { BLACK-BACKED WOODPECKER } \\
\text { PILEATED WOODPECKER }\end{array}$} & & 4 & & & & & & & & \\
\hline & 2 & 2 & 4 & 2 & & 4 & 1 & & 1 & 1 \\
\hline & 1 & 2 & 4 & 2 & & 2 & & & 4 & \\
\hline & & 2 & & & & & & & & \\
\hline & & 1 & & & & & & + & & \\
\hline \multirow{5}{*}{$\begin{array}{l}\text { HORNED LARK } \\
\text { GRAY JAY } \\
\text { BLUE JAY } \\
\text { BLACK-BILLED MAGPIE } \\
\text { COMMON RAVEN }\end{array}$} & & & & & & & 3 & & & \\
\hline & & 9 & & & & & & 2 & & \\
\hline & 8 & 8 & 3 & & & 6 & 4 & 4 & 1 & 2 \\
\hline & 29 & 14 & 9 & + & 3 & 140 & 23 & 1 & 44 & 17 \\
\hline & 2 & 34 & 1 & 1 & & 18 & & 6 & 9 & 4 \\
\hline \multirow{5}{*}{$\begin{array}{l}\text { BLACK-CAPPED CHICKADEE } \\
\text { BOREAL CHICKADEE } \\
\text { RED-BREASTED NUTHATCH } \\
\text { WHITE-BREASTED NUTHATCH } \\
\text { AMERICAN ROBIN }\end{array}$} & 37 & 1 & 30 & 2 & 3 & 35 & 2 & 6 & 26 & 7 \\
\hline & & 1 & & & & & & & & \\
\hline & & & & & & 4 & & 3 & & \\
\hline & 7 & 2 & & & & & & & 1 & \\
\hline & 1 & & & & & + & & & 1 & \\
\hline \multirow{5}{*}{$\begin{array}{l}\text { BOHEMIAN WAXWING } \\
\text { CEDAR WAXWING } \\
\text { NORTHERN SHRIKE } \\
\text { EUROPEAN STARLING } \\
\text { DARK-EYED JUNCO (Slate-col.) }\end{array}$} & 58 & & 12 & & & 30 & 60 & & 74 & \\
\hline & & & & & & & & & & \\
\hline & & & & & & & 1 & & & \\
\hline & & & & & & 22 & 12 & & & \\
\hline & & & 1 & & & & & & & \\
\hline \multirow{5}{*}{$\begin{array}{l}\text { SNOW BUNTING } \\
\text { WESTERN MEADOWLARK } \\
\text { RUSTY BLACKBIRD } \\
\text { PINE GROSBEAK } \\
\text { WHITE-WINGED CROSSBILL }\end{array}$} & 28 & 13 & + & & 40 & 1417 & & & 15 & 48 \\
\hline & 1 & & & & & & & & & \\
\hline & & & & & & 5 & 1 & & & \\
\hline & 10 & & 17 & 3 & & 5 & & & 2 & 17 \\
\hline & 8 & & & & & 16 & & & & \\
\hline \multirow{5}{*}{$\begin{array}{l}\text { COMMON REDPOLL } \\
\text { HOARY REDPOLL } \\
\text { PINE SISKIN } \\
\text { EVENING GROSBEAK } \\
\text { HOUSE SPARROW }\end{array}$} & 89 & 2 & 52 & 4 & & 26 & 43 & & 46 & 32 \\
\hline & 29 & & & & & & & & 5 & \\
\hline & & & & & & & & & & \\
\hline & 1 & 50 & & & & & & & 6 & \\
\hline & 30 & & 49 & 5 & 60 & 718 & 27 & 15 & 117 & 116 \\
\hline \multirow{5}{*}{$\begin{array}{l}\text { NO. SPECIES COUNT DAY } \\
\text { NO. SPECIES COUNT PERIOD } \\
\text { NO. SPECIES TABLE } 4 \& 5 \\
\text { NO. INDIV. TABLE } 4 \& 5 \\
\text { NO. INDIVIDUALS COUNT DAY }\end{array}$} & 23 & 23 & 12 & 8 & 7 & 22 & 20 & 8 & 19 & 16 \\
\hline & 26 & 23 & 18 & 9 & 7 & 26 & 20 & 11 & 19 & 16 \\
\hline & 0 & 3 & 0 & 0 & 0 & 3 & 3 & 1 & 0 & 0 \\
\hline & 0 & 12 & 0 & 0 & 0 & 17 & 35 & 0 & 0 & 0 \\
\hline & 423 & 179 & 193| & 20 & 134 & 2764 & 268 & 41 & 363 & 308 \\
\hline
\end{tabular}


Table 3-2. SPECIES RECORDED FROM MORE THAN NINE LOCALITIES $(+=a$ species seen during the count period but not on count day).

\begin{tabular}{|c|c|c|c|c|c|c|c|c|c|c|}
\hline 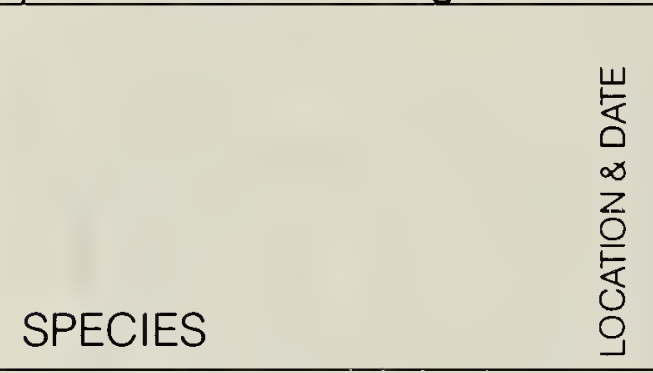 & 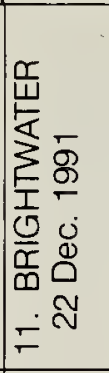 & 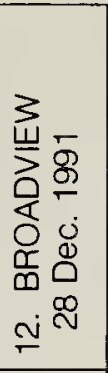 & 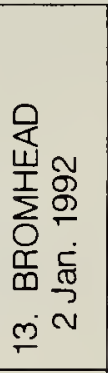 & 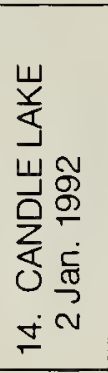 & 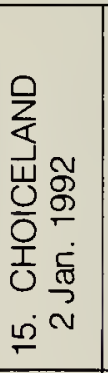 & 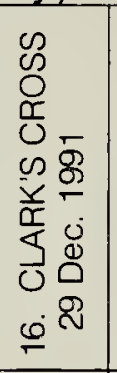 & 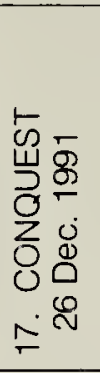 & 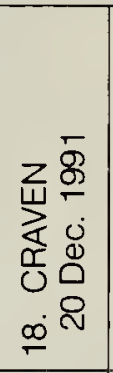 & 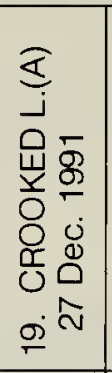 & 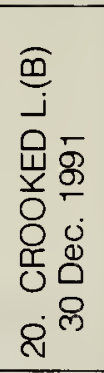 \\
\hline \multirow{5}{*}{$\begin{array}{l}\text { MALLARD } \\
\text { COMMON GOLDENEYE } \\
\text { BALD EAGLE } \\
\text { NORTHERN GOSHAWK } \\
\text { GOLDEN EAGLE }\end{array}$} & & & & & & & & & 2 & \\
\hline & & & & & & 25 & & & & \\
\hline & & & & & & & & & & 2 \\
\hline & & 1 & & & 2 & & & & 1 & \\
\hline & 1 & & 2 & & & & & & 4 & \\
\hline \multirow{5}{*}{$\begin{array}{l}\text { MERLIN } \\
\text { PRAIRIE FALCON } \\
\text { GRAY PARTRIDGE } \\
\text { RING-NECKED PHEASANT } \\
\text { RUFFED GROUSE }\end{array}$} & & & & & & & & & & \\
\hline & & & 1 & & & & & & & \\
\hline & 19 & 11 & & & & 51 & 13 & & 13 & 10 \\
\hline & & & 3 & & & & & & & \\
\hline & & 1 & & 4 & 3 & & & 3 & 1 & \\
\hline \multirow{5}{*}{$\begin{array}{l}\text { SHARP-TAILED GROUSE } \\
\text { ROCK DOVE } \\
\text { GREAT HORNED OWL } \\
\text { SNOWY OWL } \\
\text { NORTHERN HAWK OWL }\end{array}$} & 1 & 61 & 42 & & 17 & 25 & & & 134 & 14 \\
\hline & & 6 & & & 48 & 50 & 40 & 4 & 3 & \\
\hline & & & 4 & & 1 & & 1 & & & \\
\hline & 2 & + & 1 & & 1 & 3 & & & & 1 \\
\hline & & & & 2 & 2 & & & & & \\
\hline \multirow{5}{*}{$\begin{array}{l}\text { GREAT GRAY OWL } \\
\text { DOWNY WOODPECKER } \\
\text { HAIRY WOODPECKER } \\
\text { BLACK-BACKED WOODPECKER } \\
\text { PILEATED WOODPECKER }\end{array}$} & & & & 1 & & & & & & \\
\hline & 1 & 1 & & 3 & 7 & 1 & 1 & 2 & 3 & 5 \\
\hline & 3 & 1 & & 2 & 3 & & & 1 & 4 & 1 \\
\hline & & & & 1 & 1 & & & & & \\
\hline & & & & 3 & 2 & & & & & \\
\hline \multirow{5}{*}{$\begin{array}{l}\text { HORNED LARK } \\
\text { GRAY JAY } \\
\text { BLUE JAY } \\
\text { BLACK-BILLED MAGPIE } \\
\text { COMMON RAVEN }\end{array}$} & & & 34 & & & & & & & \\
\hline & & & & 26 & 19 & & & & & \\
\hline & & 1 & & 2 & 13 & & 1 & 11 & 7 & 5 \\
\hline & 16 & 30 & 4 & 14 & 28 & 115 & 20 & 50 & 47 & 6 \\
\hline & 4 & 4 & & 49 & 62 & 3 & & & & 1 \\
\hline \multirow{5}{*}{$\begin{array}{l}\text { BLACK-CAPPED CHICKADEE } \\
\text { BOREAL CHICKADEE } \\
\text { RED-BREASTED NUTHATCH } \\
\text { WHITE-BREASTED NUTHATCH } \\
\text { AMERICAN ROBIN }\end{array}$} & 8 & 22 & & 50 & 11 & 15 & 11 & 31 & 44 & 33 \\
\hline & & & & 21 & 2 & & & & & \\
\hline & & & & 10 & 1 & & & & & \\
\hline & & 3 & & 1 & & & & & 3 & 6 \\
\hline & & & & & & & & & & \\
\hline \multirow{5}{*}{$\begin{array}{l}\text { BOHEMIAN WAXWING } \\
\text { CEDAR WAXWING } \\
\text { NORTHERN SHRIKE } \\
\text { EUROPEAN STARLING } \\
\text { DARK-EYED JUNCO (Slate-Col.) }\end{array}$} & 46 & 85 & & & 18 & 81 & & 30 & 56 & 23 \\
\hline & & & & & & & & & & 34 \\
\hline & & + & & & & & 1 & 3 & 1 & \\
\hline & & 7 & 17 & & & 32 & 20 & & & \\
\hline & 1 & & & & & & & & & 9 \\
\hline \multirow{5}{*}{$\begin{array}{l}\text { SNOW BUNTING } \\
\text { WESTERN MEADOWLARK } \\
\text { RUSTY BLACKBIRD } \\
\text { PINE GROSBEAK } \\
\text { WHITE-WINGED CROSSBILL }\end{array}$} & 1 & 15 & 294 & & 172 & 108 & 30 & & & \\
\hline & & & & & & & & & & \\
\hline & 4 & & & & & & & & & \\
\hline & 13 & 21 & & 13 & 32 & 7 & 5 & 16 & 17 & \\
\hline & & & & 1 & 14 & & & & & \\
\hline \multirow{5}{*}{$\begin{array}{l}\text { COMMON REDPOLL } \\
\text { HOARY REDPOLL } \\
\text { PINE SISKIN } \\
\text { EVENING GROSBEAK } \\
\text { HOUSE SPARROW }\end{array}$} & 141 & 40 & 326 & 34 & 112 & 2 & 49 & 176 & 138 & 55 \\
\hline & & & 3 & 1 & 2 & & & & & \\
\hline & & & & & & & & & & 4 \\
\hline & & & & 95 & 8 & & & & & 6 \\
\hline & 10 & 178 & 140 & & 68 & 954 & 66 & 187 & 20 & 31 \\
\hline \multirow{5}{*}{$\begin{array}{l}\text { NO. SPECIES COUNT DAY } \\
\text { NO. SPECIES COUNT PERIOD } \\
\text { NO. SPECIES TABLE } 4 \& 5 \\
\text { NO. INDIV. TABLE } 4 \text { \& } 5 \\
\text { NO. INDIVIDUALS COUNT DAY }\end{array}$} & 16 & 18 & 14 & 21 & 28 & 15 & 13 & 12 & 18 & 19 \\
\hline & 16 & 20 & 14 & 21 & 28 & 15 & 13 & 12 & 18 & 19 \\
\hline & 0 & 0 & 1 & 1 & 2 & 1 & 0 & 1 & 0 & 1 \\
\hline & 0 & 0 & 33 & 1 & 2 & 1 & 0 & 8 & 0 & 2 \\
\hline & 271 & 488 & 904 & 334 & 651 & 1473 & 258 & 522 & 498 & 248 \\
\hline
\end{tabular}


Table 3-3. SPECIES RECORDED FROM MORE THAN NINE LOCALITIES $(+=a$ species seen during the count period but not on count day).

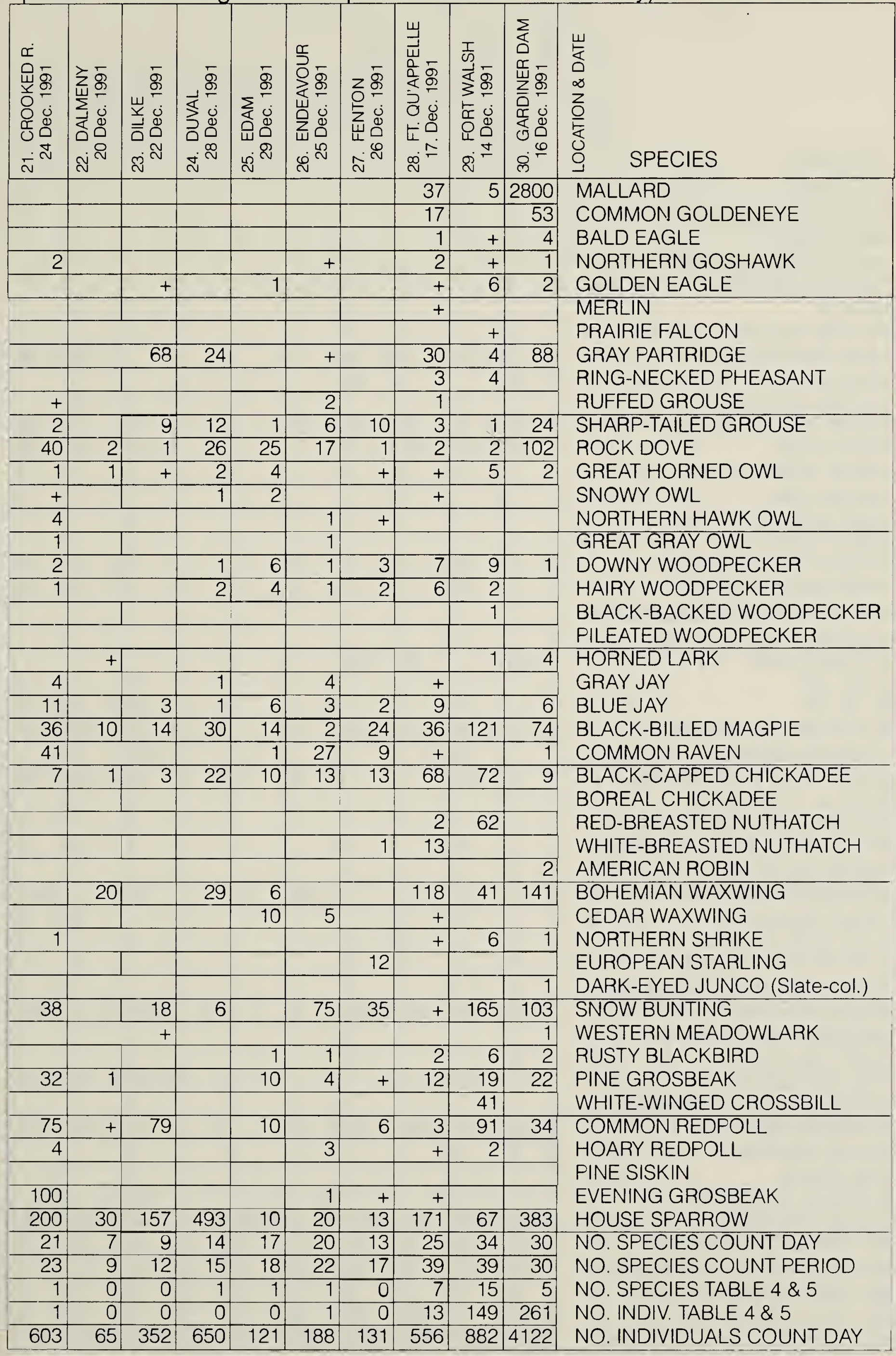


Table 3-4. SPECIES RECORDED FROM MORE THAN NINE LOCALITIES $(+=a$ species seen during the count period but not on count day).

SPECIES

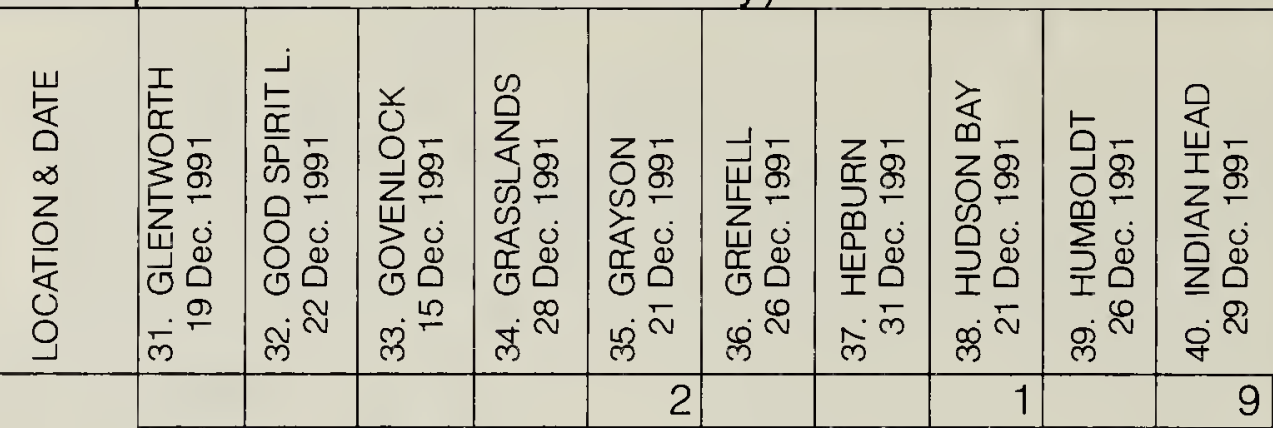

\section{MALLARD}

COMMON GOLDENEYE

BALD EAGLE

NORTHERN GOSHAWK

GOLDEN EAGLE

MERLIN

PRAIRIE FALCON

GRAY PARTRIDGE

RING-NECKED PHEASANT

RUFFED GROUSE

SHARP-TAILED GROUSE

ROCK DOVE

GREAT HORNED OWL

SNOWY OWL

NORTHERN HAWK OWL

GREAT GRAY OWL

DOWNY WOODPECKER

HAIRY WOODPECKER

BLACK-BACKED WOODPECKER

PILEATED WOODPECKER

HORNED LARK

GRAY JAY

BLUE JAY

BLACK-BILLED MAGPIE

COMMON RAVEN

BLACK-CAPPED CHICKADEE BOREAL CHICKADEE

RED-BREASTED NUTHATCH

WHITE-BREASTED NUTHATCH

AMERICAN ROBIN

BOHEMIAN WAXWING

CEDAR WAXWING

NORTHERN SHRIKE

EUROPEAN STARLING

DARK-EYED JUNCO (Slate-col.)

SNOW BUNTING

WESTERN MEADOWLARK

RUSTY BLACKBIRD

PINE GROSBEAK

WHITE-WINGED CROSSBILL

COMMON REDPOLL

HOARY REDPOLL

PINE SISKIN

EVENING GROSBEAK

HOUSE SPARROW

NO. SPECIES COUNT DAY

NO. SPECIES COUNT PERIOD

NO. SPECIES TABLE $4 \& 5$

NO. INDIV. TABLE $4 \& 5$

NO. INDIVIDUALS COUNT DAY 
Table 3-5. SPECIES RECORDED FROM MORE THAN NINE LOCALITIES $(+=\mathrm{a}$ species seen during the count period but not on count day).

\begin{tabular}{|c|c|c|c|c|c|c|c|c|c|c|}
\hline 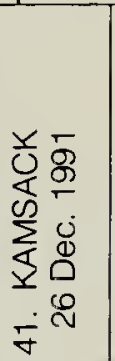 & 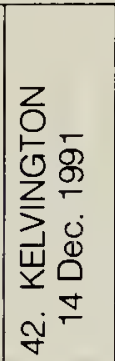 & 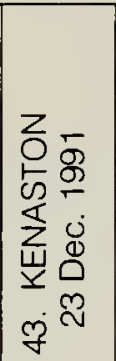 & 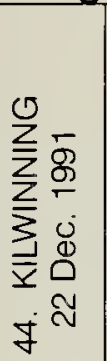 & 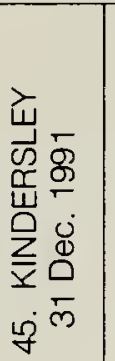 & 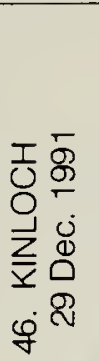 & 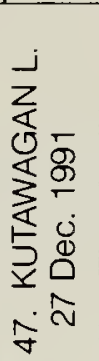 & 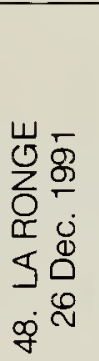 & 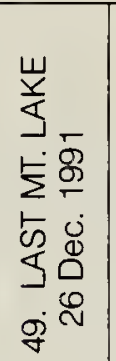 & 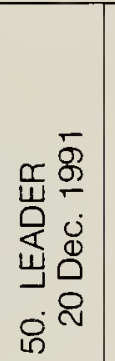 & 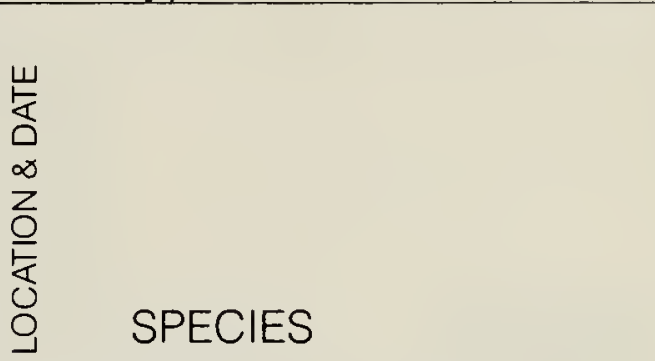 \\
\hline & & & & & & & & & & \multirow{5}{*}{$\begin{array}{l}\text { MALLARD } \\
\text { COMMON GOLDENEYE } \\
\text { BALD EAGLE } \\
\text { NORTHERN GOSHAWK } \\
\text { GOLDEN EAGLE }\end{array}$} \\
\hline & & & & & & & & & & \\
\hline & & & & & & & & & 2 & \\
\hline & & & & & & & & & & \\
\hline & & + & & & & & & 2 & 2 & \\
\hline & & & & & & & & & & \multirow{5}{*}{$\begin{array}{l}\text { MERLIN } \\
\text { PRAIRIE FALCON } \\
\text { GRAY PARTRIDGE } \\
\text { RING-NECKED PHEASANT } \\
\text { RUFFED GROUSE }\end{array}$} \\
\hline & & & & & & & & & & \\
\hline 40 & 12 & 75 & & 8 & + & 39 & & 137 & 21 & \\
\hline & & & & & & & & & 19 & \\
\hline & & & & & + & & + & & & \\
\hline 8 & & + & 3 & & 3 & & & 17 & 1 & \multirow{5}{*}{$\begin{array}{l}\text { SHARP-TAILED GROUSE } \\
\text { ROCK DOVE } \\
\text { GREAT HORNED OWL } \\
\text { SNOWY OWL } \\
\text { NORTHERN HAWK OWL }\end{array}$} \\
\hline & & 18 & 1 & & + & 1 & & 106 & & \\
\hline 1 & & & 1 & & + & & & 1 & 1 & \\
\hline & & + & & 7 & & 3 & & 8 & & \\
\hline & & & & & 1 & & & & & \\
\hline 1 & & & & & & & & & & \multirow{5}{*}{$\begin{array}{l}\text { GREAT GRAY OWL } \\
\text { DOWNY WOODPECKER } \\
\text { HAIRY WOODPECKER } \\
\text { BLACK-BACKED WOODPECKER } \\
\text { PILEATED WOODPECKER }\end{array}$} \\
\hline 3 & 2 & + & 1 & & 1 & & + & 1 & 1 & \\
\hline 6 & 3 & + & 1 & & 2 & & 1 & & & \\
\hline & & & & & & & & & & \\
\hline 7 & & & & & 1 & & 1 & & 1 & \\
\hline & & & 3 & & & & & 2 & & \multirow{10}{*}{$\begin{array}{l}\text { HORNED LARK } \\
\text { GRAY JAY } \\
\text { BLUE JAY } \\
\text { BLACK-BILLED MAGPIE } \\
\text { COMMON RAVEN } \\
\text { BLACK-CAPPED CHICKADEE } \\
\text { BOREAL CHICKADEE } \\
\text { RED-BREASTED NUTHATCH } \\
\text { WHITE-BREASTED NUTHATCH } \\
\text { AMERICAN ROBIN }\end{array}$} \\
\hline & & & & & 1 & & 13 & & & \\
\hline 30 & 10 & 1 & 2 & & 21 & 1 & 5 & & & \\
\hline 19 & 9 & 13 & 47 & 8 & 11 & 43 & 3 & 97 & 48 & \\
\hline 28 & 8 & + & 4 & & 19 & & 106 & & & \\
\hline 62 & 20 & 2 & 23 & & 26 & & 9 & 1 & 2 & \\
\hline 4 & & & 1 & & & & 2 & & & \\
\hline 11 & & & 1 & & & & 1 & & & \\
\hline 4 & & & & & 1 & & & & & \\
\hline 4 & & & & & & & & & & \\
\hline 280 & 12 & & & & 1 & & & & 2 & \multirow{5}{*}{$\begin{array}{l}\text { BOHEMIAN WAXWING } \\
\text { CEDAR WAXWING } \\
\text { NORTHERN SHRIKE } \\
\text { EUROPEAN STARLING } \\
\text { DARK-EYED JUNCO (Slate-col.) }\end{array}$} \\
\hline 20 & & & & & & & & & & \\
\hline & & + & 1 & & & & & & & \\
\hline & & & & & & & & 2 & & \\
\hline 16 & & & & & & & + & & & \\
\hline 100 & 100 & 254 & 370 & & 12 & 165 & & 533 & & \multirow{5}{*}{$\begin{array}{l}\text { SNOW BUNTING } \\
\text { WESTERN MEADOWLARK } \\
\text { RUSTY BLACKBIRD } \\
\text { PINE GROSBEAK } \\
\text { WHITE-WINGED CROSSBILL }\end{array}$} \\
\hline & & & & & & & & 1 & & \\
\hline & & & & & & & & & & \\
\hline 222 & 6 & 27 & 6 & & 11 & 1 & 23 & 4 & & \\
\hline & & & & & & & & & & \\
\hline 71 & 30 & 1 & 97 & & 19 & 8 & 59 & 71 & 20 & \multirow{5}{*}{$\begin{array}{l}\text { COMMON REDPOLL } \\
\text { HOARY REDPOLL } \\
\text { PINE SISKIN } \\
\text { EVENING GROSBEAK } \\
\text { HOUSE SPARROW }\end{array}$} \\
\hline & & & 2 & & 6 & & 7 & & 10 & \\
\hline 30 & & & & & & & & & & \\
\hline 429 & 50 & & & & 20 & & 15 & & & \\
\hline 186 & 20 & 17 & 80 & 56 & 34 & 125 & & 553 & 62 & \\
\hline 26 & 13 & 9 & 18 & 4 & 18 & 9 & 13 & 19 & 14 & NO. SPECIES COUNT DAY \\
\hline 27 & 13 & 16 & 18 & 4 & 22 & 9 & 16 & 19 & 14 & NO. SPECIES COUNT PERIOD \\
\hline 3 & 0 & 0 & 0 & 0 & 0 & 0 & 0 & 3 & 0 & NO. SPECIES TABLE $4 \& 5$ \\
\hline 19 & 0 & 0 & 0 & 0 & 0 & 0 & 0 & 3 & 0 & NO. INDIV. TABLE $4 \& 5$ \\
\hline 1601 & 282 & 408 & 644 & 79 & 190 & 386 & 245 & 1542 & 192 & NO. INDIVIDUALS COUNT DAY \\
\hline
\end{tabular}


Table 3-6. SPECIES RECORDED FROM MORE THAN NINE LOCALITIES $(+=a$ species seen during the count period but not on count day).

SPECIES
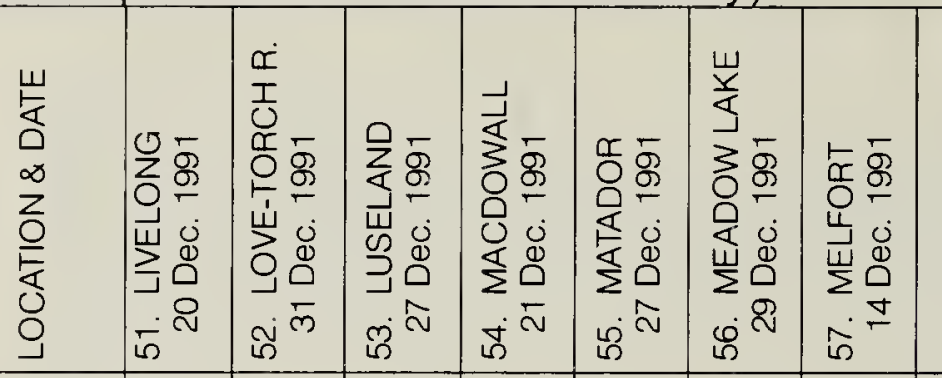

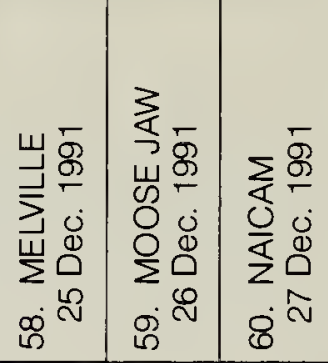

MALLARD

COMMON GOLDENEYE

BALD EAGLE

NORTHERN GOSHAWK

GOLDEN EAGLE

MERLIN

PRAIRIE FALCON

GRAY PARTRIDGE

RING-NECKED PHEASANT

RUFFED GROUSE

SHARP-TAILED GROUSE

ROCK DOVE

GREAT HORNED OWL

SNOWY OWL

NORTHERN HAWK OWL

GREAT GRAY OWL

DOWNY WOODPECKER

HAIRY WOODPECKER

BLACK-BACKED WOODPECKER

PILEATED WOODPECKER

HORNED LARK

GRAY JAY

BLUE JAY

BLACK-BILLED MAGPIE

COMMON RAVEN

BLACK-CAPPED CHICKADEE

BOREAL CHICKADEE

RED-BREASTED NUTHATCH

WHITE-BREASTED NUTHATCH

AMERICAN ROBIN

BOHEMIAN WAXWING

CEDAR WAXWING

NORTHERN SHRIKE

EUROPEAN STARLING

DARK-EYED JUNCO (Slate-col.)

SNOW BUNTING

WESTERN MEADOWLARK

RUSTY BLACKBIRD

PINE GROSBEAK

WHITE-WINGED CROSSBILL

COMMON REDPOLL

HOARY REDPOLL

PINE SISKIN

EVENING GROSBEAK

HOUSE SPARROW

NO. SPECIES COUNT DAY

NO. SPECIES COUNT PERIOD

NO. SPECIES TABLE 4 \& 5

NO. INDIV. TABLE 4 \& 5

NO. INDIVIDUALS COUNT DAY

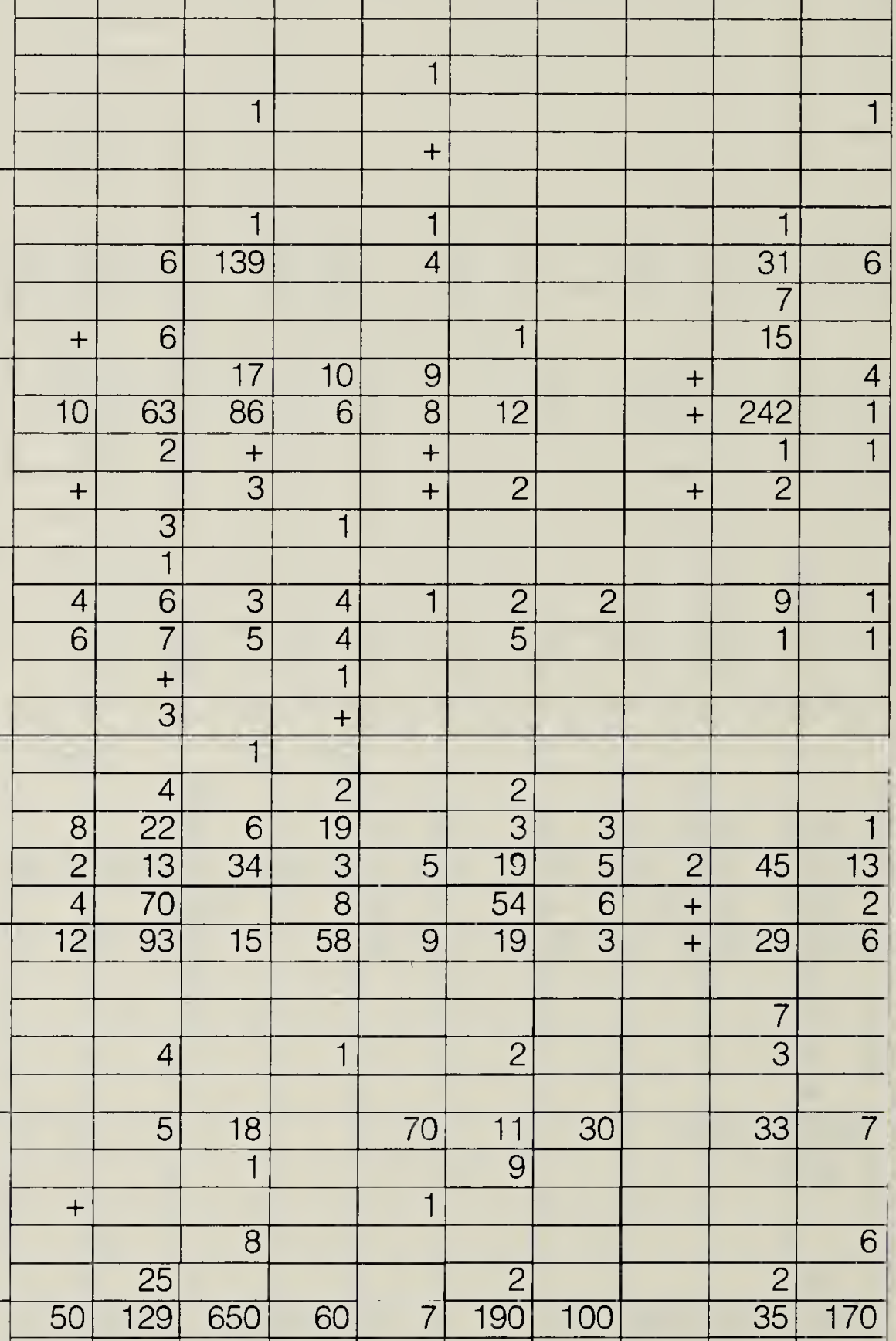

\begin{tabular}{|r|r|r|r|r|r|r|r|r|r}
\hline & & & & & & & & & \\
\hline & & & & & & & & & \\
\hline 20 & 41 & + & 9 & + & 23 & & 8 & 4 & 16 \\
\hline 30 & 385 & 52 & 24 & & 44 & & 8 & 40 & 2 \\
\hline & & & 6 & 40 & & & & & \\
\hline & 25 & & & & 17 & & & & \\
\hline 20 & 306 & & 121 & & 102 & & & + & \\
\hline 50 & 80 & 369 & 30 & & 299 & 100 & 15 & 475 & 244 \\
\hline 12 & 25 & 19 & 18 & 12 & 22 & 8 & 4 & 22 & 17 \\
\hline 16 & 26 & 21 & 21 & 16 & 22 & 8 & 9 & 24 & 17 \\
\hline 1 & 2 & 1 & 1 & 0 & 2 & 0 & 0 & 4 & 0 \\
\hline 0 & 4 & 1 & 0 & 0 & 8 & 0 & 0 & 5 & 0 \\
\hline 216 & 1303 & 1410 & 367 & 156 & 826 & 249 & 33 & 987 & 482 \\
\hline
\end{tabular}


Table 3-7. SPECIES RECORDED FROM MORE THAN NINE LOCALITIES $(+=\mathrm{a}$ species seen during the count period but not on count day).

\begin{tabular}{|c|c|c|c|c|c|c|c|c|c|c|}
\hline 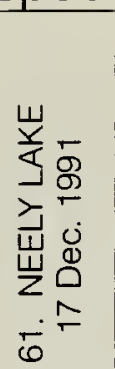 & 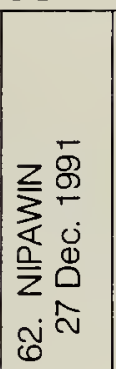 & 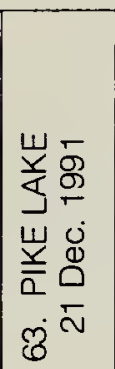 & 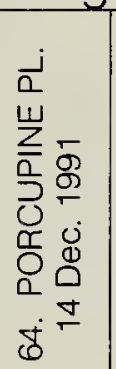 & 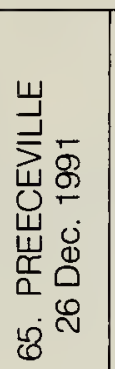 & 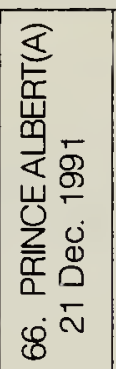 & 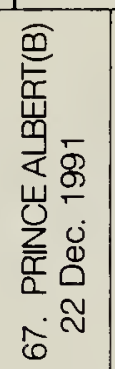 & 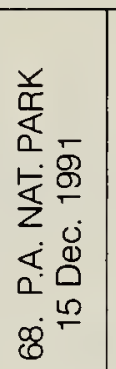 & 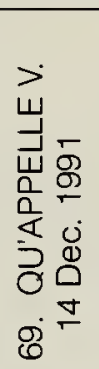 & 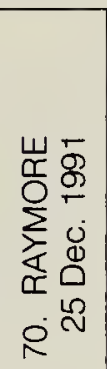 & 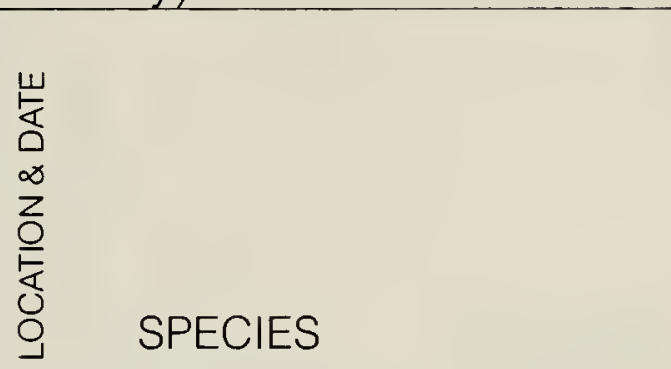 \\
\hline & & & & & & & & & & \multirow{5}{*}{$\begin{array}{l}\text { MALLARD } \\
\text { COMMON GOLDENEYE } \\
\text { BALD EAGLE } \\
\text { NORTHERN GOSHAWK } \\
\text { GOLDEN EAGLE }\end{array}$} \\
\hline & & & & & & & & 1 & & \\
\hline & & & 1 & & & & & 1 & + & \\
\hline & & 1 & + & & & & + & & 2 & \\
\hline & & & & & & & & $\overline{2}$ & & \\
\hline & & & & & & + & & & 1 & \multirow{2}{*}{$\begin{array}{l}\text { MERLIN } \\
\text { PRAIRIE FALCON }\end{array}$} \\
\hline & & & & & & & & & & \\
\hline 7 & & 31 & 5 & & & & & 19 & 17 & \multirow{2}{*}{$\begin{array}{l}\text { GRAY PARTRIDGE } \\
\text { RING-NECKED PHEASANT }\end{array}$} \\
\hline & & & & & & & & & & \\
\hline+ & & & 1 & & & & 1 & & 4 & RUFFED GROUSE \\
\hline 33 & & 150 & & 5 & & & & 54 & 116 & SHARP-TAILED GROUSE \\
\hline 4 & & 15 & & 12 & & 333 & & 2 & 211 & \multirow{4}{*}{$\begin{array}{l}\text { ROCK DOVE } \\
\text { GREAT HORNED OWL } \\
\text { SNOWY OWL } \\
\text { NORTHERN HAWK OWL }\end{array}$} \\
\hline+ & & & 1 & & & 1 & + & 3 & 4 & \\
\hline & & & & & & & & & 1 & \\
\hline 1 & & & 2 & & & & & & & \\
\hline & & & & & & & + & & & \multirow{5}{*}{$\begin{array}{l}\text { GREAT GRAY OWL } \\
\text { DOWNY WOODPECKER } \\
\text { HAIRY WOODPECKER } \\
\text { BLACK-BACKED WOODPECKER } \\
\text { PILEATED WOODPECKER }\end{array}$} \\
\hline 6 & 2 & 4 & 2 & 1 & 2 & 4 & 2 & & 2 & \\
\hline 5 & 1 & 8 & 4 & 1 & 2 & 4 & 4 & 2 & 3 & \\
\hline & & & & & & & 1 & & & \\
\hline 2 & & & & & 1 & & 2 & & + & \\
\hline & & & & & & & & 6 & 1 & HORNED LARK \\
\hline 2 & & & 2 & & & & 3 & & & GRAY JAY \\
\hline 18 & 4 & 18 & 24 & 2 & 2 & 11 & & 4 & 4 & BLUE JAY \\
\hline 6 & + & 133 & 35 & 17 & 2 & 42 & 9 & 64 & 72 & BLACK-BILLED MAGPIE \\
\hline 11 & 51 & 21 & 146 & 9 & & 142 & 18 & & 1 & COMMON RAVEN \\
\hline 36 & 19 & 134 & 50 & 8 & 6 & 36 & 41 & 6 & 11 & \multirow{5}{*}{$\begin{array}{l}\text { BLACK-CAPPED CHICKADEE } \\
\text { BOREAL CHICKADEE } \\
\text { RED-BREASTED NUTHATCH } \\
\text { WHITE-BREASTED NUTHATCH } \\
\text { AMERICAN ROBIN }\end{array}$} \\
\hline & & & & & & & 4 & & & \\
\hline 1 & & 3 & & & & & 1 & & + & \\
\hline 3 & 1 & 6 & 4 & & 2 & 1 & 1 & & & \\
\hline & + & 3 & & & & + & & & 1 & \\
\hline & + & 362 & 15 & & 8 & 417 & & 31 & + & BOHEMIAN WAXWING \\
\hline & & & & & & & & & & CEDAR WAXWING \\
\hline & & 3 & & & & 2 & & 1 & 2 & NORTHERN SHRIKE \\
\hline & & 19 & & & & & & 1 & 4 & EUROPEAN STARLING \\
\hline+ & + & 1 & & & & & & & 2 & DARK-EYED JUNCO (Slate-col.) \\
\hline 33 & & 170 & 15 & 17 & & 6 & & 269 & 362 & SNOW BUNTING \\
\hline & & & & & & & & & 1 & WESTERN MEADOWLARK \\
\hline & & & & & & & & & + & RUSTY BLACKBIRD \\
\hline 53 & 4 & 158 & 23 & 27 & 10 & 9 & & 23 & 17 & PINE GROSBEAK \\
\hline & & 4 & & & & & 18 & & 4 & WHITE-WINGED CROSSBILL \\
\hline 11 & + & 279 & 2 & 46 & 8 & 1 & 7 & 79 & 36 & COMMON REDPOLL \\
\hline 8 & & 3 & & & & & & & 2 & HOARY REDPOLL \\
\hline & & & & & & 14 & & & & PINE SISKIN \\
\hline 49 & 22 & 2 & 88 & 22 & 20 & 14 & & & + & EVENING GROSBEAK \\
\hline 94 & & 177 & 103 & 10 & & 189 & & 217 & 463 & HOUSE SPARROW \\
\hline 20 & 8 & 25 & 19 & 13 & 11 & 19 & 14 & 21 & 27 & NO. SPECIES COUNT DAY \\
\hline 23 & 13 & 25 & 20 & 13 & 11 & 22 & 18 & 21 & 35 & NO. SPECIES COUNT PERIOD \\
\hline 0 & 0 & 1 & 0 & 0 & 0 & 2 & 1 & 2 & 3 & NO. SPECIES TABLE 4 \& 5 \\
\hline 0 & 0 & 2 & 0 & 0 & 0 & 1 & 0 & 2 & 1 & NO. INDIV. TABLE $4 \& 5$ \\
\hline 383 & 104 & 1707 & 523 & 177 & 63 & 1227 & 112 & 787 & 1345 & NO. INDIVIDUALS COUNT DAY \\
\hline
\end{tabular}


Table 3-8. SPECIES RECORDED FROM MORE THAN NINE LOCALITIES $(+=a$ species seen during the count period but not on count day).

\section{SPECIES \\ MALLARD}

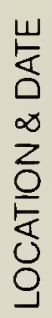

COMMON GOLDENEYE

BALD EAGLE

NORTHERN GOSHAWK

GOLDEN EAGLE

MERLIN

PRAIRIE FALCON

GRAY PARTRIDGE

RING-NECKED PHEASANT

RUFFED GROUSE

SHARP-TAILED GROUSE

ROCK DOVE

GREAT HORNED OWL

SNOWY OWL

NORTHERN HAWK OWL

GREAT GRAY OWL

DOWNY WOODPECKER

HAIRY WOODPECKER

BLACK-BACKED WOODPECKER

PILEATED WOODPECKER

HORNED LARK

GRAY JAY

BLUE JAY

BLACK-BILLED MAGPIE

COMMON RAVEN

BLACK-CAPPED CHICKADEE

BOREAL CHICKADEE

RED-BREASTED NUTHATCH

WHITE-BREASTED NUTHATCH

AMERICAN ROBIN

BOHEMIAN WAXWING

ICEDAR WAXWING

NORTHERN SHRIKE

EUROPEAN STARLING

DARK-EYED JUNCO (Slate-col.)

SNOW BUNTING

WESTERN MEADOWLARK

RUSTY BLACKBIRD

PINE GROSBEAK

WHITE-WINGED CROSSBILL

COMMON REDPOLL

HOARY REDPOLL

PINE SISKIN

EVENING GROSBEAK

HOUSE SPARROW

NO. SPECIES COUNT DAY

NO. SPECIES COUNT PERIOD

NO. SPECIES TABLE $4 \& 5$

NO. INDIV. TABLE $4 \& 5$

NO. INDIVIDUALS COUNT DAY

\begin{tabular}{|c|c|c|c|c|c|c|c|c|c|}
\hline 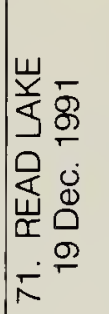 & 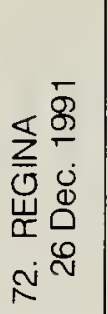 & 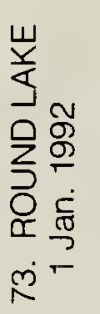 & 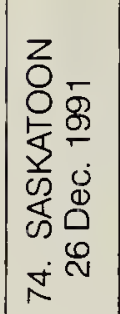 & 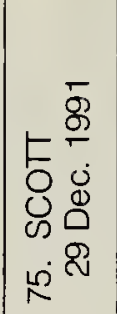 & 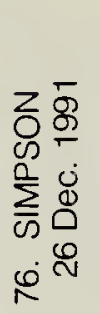 & 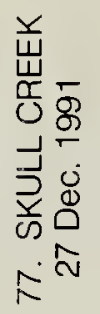 & 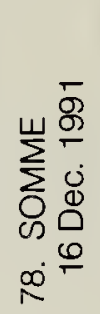 & 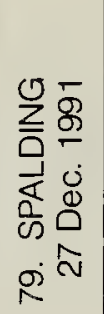 & 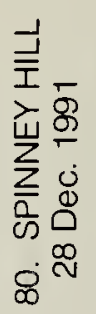 \\
\hline & 96 & + & 6 & & & & & & \\
\hline & & & 70 & & & & & & \\
\hline & & & & & & & & & \\
\hline & + & & 1 & & & 1 & + & & \\
\hline & & + & & & & + & & & \\
\hline & 1 & & 5 & & & 1 & & & \\
\hline & & & & & & & & & \\
\hline & 93 & 9 & 75 & 113 & 27 & & & 19 & \\
\hline & 1 & & & & & + & & & \\
\hline & & 4 & 4 & & & 2 & 2 & 5 & \\
\hline & 7 & $\overline{8}$ & 70 & & & 18 & 2 & & 42 \\
\hline & 968 & & 2023 & 79 & & 2 & 16 & 7 & 25 \\
\hline & 7 & + & 6 & 2 & 2 & 3 & + & & \\
\hline & 7 & & 1 & 1 & & -1 & 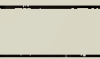 & & \\
\hline & & & & & & & 2 & & \\
\hline & & & & & & & & & \\
\hline 2 & 7 & 8 & 37 & 3 & 2 & 11 & 3 & 2 & 1 \\
\hline & 4 & 7 & 36 & 3 & 2 & 2 & 1 & 1 & 3 \\
\hline & & & + & & & & + & & \\
\hline & & & & & & & + & & 1 \\
\hline & & & & & & 10 & & & \\
\hline 4 & & & & & & & 4 & & \\
\hline & 3 & 4 & 86 & 2 & 9 & 1 & 49 & 2 & 8 \\
\hline & 79 & 5 & 648 & 55 & 7 & 65 & 38 & 9 & 58 \\
\hline 2 & & 2 & 7 & & & & 78 & 1 & 2 \\
\hline & 37 & 35 & 390 & 21 & 17 & 70 & 28 & 6 & 30 \\
\hline & & & & & & & 8 & & \\
\hline & 8 & & 58 & 3 & & & & & \\
\hline & 3 & 6 & 10 & & & 1 & 1 & & \\
\hline & 6 & & 1 & & & & & & \\
\hline & 26 & 60 & 1932 & 10 & 23 & 20 & + & & \\
\hline & 27 & & 22 & & & & & & \\
\hline & & & & 1 & & 3 & + & & \\
\hline & & & 11 & 13 & & 5 & & 5 & \\
\hline & 7 & 3 & 7 & & & & 1 & & \\
\hline & 12 & & 5 & 445 & 37 & + & + & 1 & \\
\hline & 5 & & 1 & & & 5 & & & \\
\hline & 2 & 10 & $\frac{1}{110}$ & 11 & & $\frac{3}{1}$ & 20 & 4 & 4 \\
\hline & 67 & & 168 & 45 & & & & & $\overline{12}$ \\
\hline 3 & 13 & 38 & 688 & 65 & 3 & 60 & 20 & 24 & 453 \\
\hline & & 22 & 1 & 1 & & & 4 & & 1 \\
\hline & & & 16 & & & & & & \\
\hline & & 20 & 7 & & 7 & & 20 & & $\overline{6}$ \\
\hline & 1209 & 50 & 3759 & 418 & 30 & 202 & 177 & 50 & 58 \\
\hline 4 & 29 & 17 & 41 & 19 & 12 & 23 & 20 & 15 & 17 \\
\hline 4 & 31 & 21 & 42 & 19 & 12 & 26 & 27 & 15 & 17 \\
\hline 0 & 9 & 1 & 10 & 0 & 0 & 3 & 1 & 1 & 2 \\
\hline 0 & 456 & 0 & 34 & 0 & 0 & 54 & 1 & 7 & $\overline{2}$ \\
\hline 11 & 3146 & 291 & 10295 & 1291 & 166 & 537 & 475 & 136 & 706 \\
\hline
\end{tabular}


Table 3-9. SPECIES RECORDED FROM MORE THAN NINE LOCALITIES $(+=a$ species seen during the count period but not on count day).

\begin{tabular}{|c|c|c|c|c|c|c|c|c|c|c|}
\hline 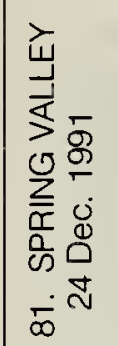 & 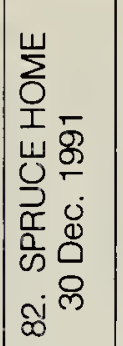 & 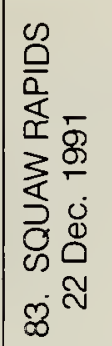 & 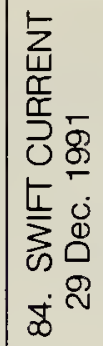 & 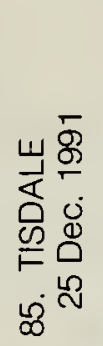 & 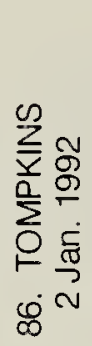 & 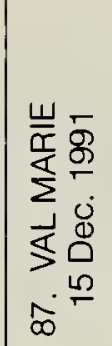 & 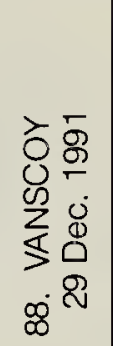 & 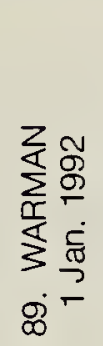 & 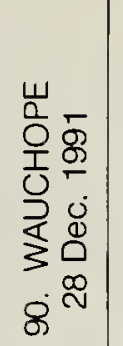 & $\begin{array}{ll}\text { 崖 } & \\
\infty & \\
\infty & \\
2 & \\
\text { Oे } & \\
& \\
0 & \text { SPECIES }\end{array}$ \\
\hline & & 6 & + & & & 15 & & & & \multirow{5}{*}{$\begin{array}{l}\text { MALLARD } \\
\text { COMMON GOLDENEYE } \\
\text { BALD EAGLE } \\
\text { NORTHERN GOSHAWK } \\
\text { GOLDEN EAGLE }\end{array}$} \\
\hline & & 26 & \pm & & & & & & & \\
\hline & & 3 & & & & & & & & \\
\hline 1 & & 1 & & & & & & & & \\
\hline 2 & & & & & 2 & 1 & & & & \\
\hline & & & & & & 1 & & & & MERLIN \\
\hline 1 & & & & & & & & & & PRAIRIE FALCON \\
\hline 92 & & & 20 & $\overline{6}$ & & + & 18 & 69 & 18 & GRAY PARTRIDGE \\
\hline & & & & & & 6 & & & 1 & RING-NECKED PHEASANT \\
\hline & 1 & 5 & & 1 & & & & & 2 & RUFFED GROUSE \\
\hline 26 & & 5 & & & 5 & 12 & & 11 & 68 & SHARP-TAILED GROUSE \\
\hline 23 & & & 118 & & & 13 & 50 & 27 & 16 & ROCK DOVE \\
\hline 3 & & $\overline{2}$ & & & & 10 & 1 & 1 & & GREAT HORNED OWL \\
\hline 2 & & & & 1 & & & 2 & 1 & & SNOWY OWL \\
\hline & & 3 & & & & & & & & NORTHERN HAWK OWL \\
\hline & & + & & & & & & & & GREAT GRAY OWL \\
\hline & 2 & 3 & 1 & 3 & & 2 & 1 & 2 & 7 & DOWNY WOODPECKER \\
\hline & 2 & 8 & & 5 & & & 1 & 2 & 4 & HAIRY WOODPECKE \\
\hline & & 2 & & & & & & & & BLACK-BACKED WOODPECKER \\
\hline & & 1 & & & & & & & & PILEATED WOODPECKER \\
\hline 52 & & & & & & 410 & & & & HORNED LARK \\
\hline & & 11 & \pm & & & & & & \pm & GRAY JAY \\
\hline & 4 & 12 & & 6 & & & & & & BLUE JAY \\
\hline 18 & & 37 & 36 & & $\varepsilon$ & 52 & 20 & 190 & 11 & BLACK-BILLED MAC \\
\hline & 1 & 97 & & & & & & 1 & & COM \\
\hline & 11 & 31 & & 10 & & 16 & 24 & 11 & 23 & BLACK-CAPPED CHICKADEE \\
\hline & & 15 & & & & & & & & BOREAL CHICKADEE \\
\hline & & 4 & $\varepsilon$ & & & & & & & RED-BREASTED NUTHATCH \\
\hline & 1 & 1 & 1 & & & & & & 1 & WHITE-BREASTED NUTHATCH \\
\hline & & & & & & & & & & AMERICAN ROBIN \\
\hline & & + & & & & & 10 & 18 & & BOHEMIAN WAXWING \\
\hline & & & 17 & & & & & & & CEDAR WA \\
\hline & & 1 & & & & 3 & & & 1 & NORTH \\
\hline & & 11 & 15 & & & 6 & & & & STARLING \\
\hline 1 & & & & & & & & & & DARK-EYED JUNCO (Slate-col.) \\
\hline 86 & 25 & $\overline{9}$ & t & & & 218 & 37 & 2 & & SNOW BUNTING \\
\hline+ & & & & & & & & & & WESTERN MEADOWLARK \\
\hline & & & & & & & & & & RUSTY BLACKBIRD \\
\hline & 22 & 11 & 7 & 15 & & & & 35 & & PINE GROSBEAK \\
\hline & & 6 & 18 & & & & & + & & WHITE-WINGED CROSSBILL \\
\hline 54 & 13 & 49 & 33 & & 110 & 600 & 7 & 61 & 226 & COMMON REDPOLL \\
\hline & 2 & 1 & & & & 2 & & 4 & 2 & HOARY REDPOLL \\
\hline & + & & \pm & & & & & & & PINE SISKIN \\
\hline & 12 & 83 & & 10 & & & & & & EVENING GROSBEAK \\
\hline 282 & & 25 & 592 & 20 & 75 & 280 & 80 & 401 & 94 & HOUSE SPARROW \\
\hline 15 & 13 & 38 & 15 & 10 & 5 & 18 & 12 & 17 & 14 & NOS \\
\hline 16 & 14 & 41 & 24 & 10 & 5 & 19 & 12 & 18 & 15 & NO. SPECIES COUNT PERIOD \\
\hline 1 & 0 & 12 & 6 & $\mathrm{c}$ & c & 1 & 0 & 1 & 0 & NO. SPECIES TABLE $4 \& 5$ \\
\hline & 0 & 60 & 9 & $\mathrm{c}$ & 0 & 1 & 0 & 1 & 0 & NO. INDIV. TABLE $4 \& 5$ \\
\hline 644 & 97 & 529 & 876 & 77 & 200 & 1648 & 251 & 837 & 474 & NO. INDIVIDUALS COUNT DAY \\
\hline
\end{tabular}


Table 3-10. SPECIES RECORDED FROM MORE THAN NINE LOCALITIES $(+=a$ species seen during the count period but not on count day).

MALLARD

COMMON GOLDENEYE

BALD EAGLE

NORTHERN GOSHAWK

GOLDEN EAGLE

MERLIN

PRAIRIE FALCON

GRAY PARTRIDGE

RING-NECKED PHEASANT

RUFFED GROUSE

SHARP-TAILED GROUSE

ROCK DOVE

GREAT HORNED OWL

SNOWY OWL

NORTHERN HAWK OWL

GREAT GRAY OWL

DOWNY WOODPECKER

HAIRY WOODPECKER

BLACK-BACKED WOODPECKER

PILEATED WOODPECKER

HORNED LARK

GRAY JAY

BLUE JAY

BLACK-BILLED MAGPIE

COMMON RAVEN

BLACK-CAPPED CHICKADEE

BOREAL CHICKADEE

RED-BREASTED NUTHATCH

WHITE-BREASTED NUTHATCH

AMERICAN ROBIN

BOHEMIAN WAXWING

CEDAR WAXWING

NORTHERN SHRIKE

EUROPEAN STARLING

DARK-EYED JUNCO (Slate-col.)

SNOW BUNTING

WESTERN MEADOWLARK

RUSTY BLACKBIRD

PINE GROSBEAK

WHITE-WINGED CROSSBILL

COMMON REDPOLL

HOARY REDPOLL

PINE SISKIN

EVENING GROSBEAK

HOUSE SPARROW

NO. SPECIES COUNT DAY

NO. SPECIES COUNT PERIOD

NO. SPECIES TABLE 4 \& 5

NO. INDIV. TABLE 4 \& 5

NO. INDIVIDUALS COUNT DAY
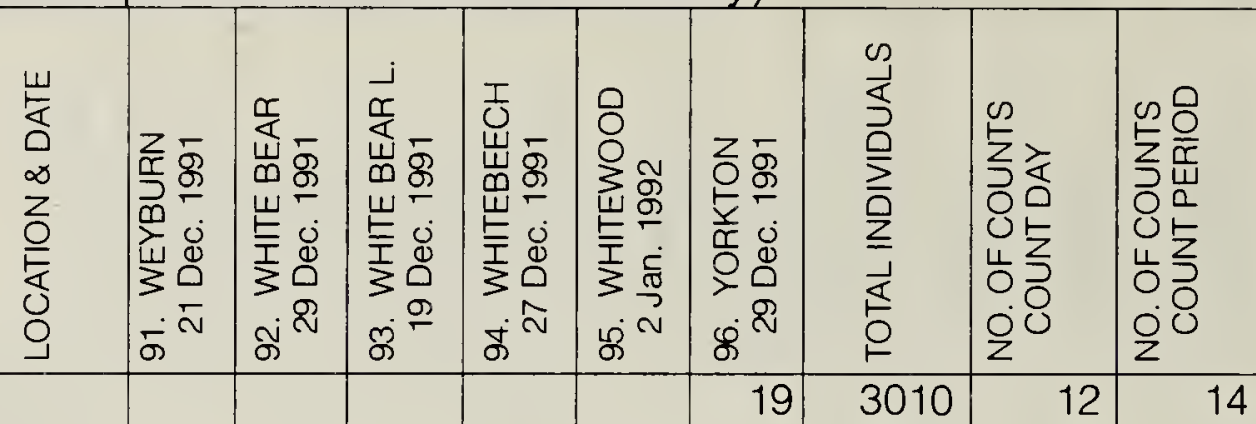

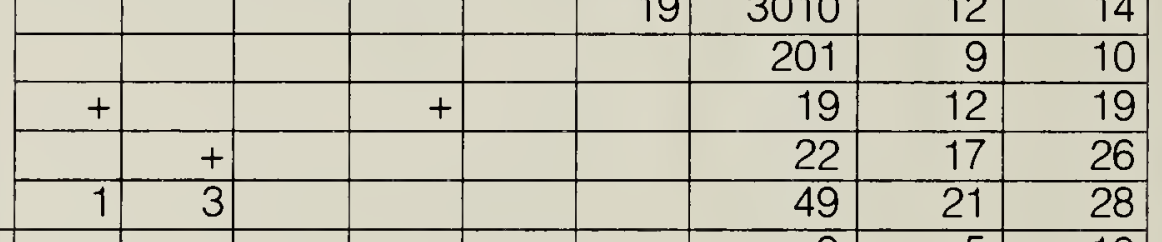

\begin{tabular}{|c|c|c|c|c|c|c|c|c|}
\hline & & & & & & & & \\
\hline+ & & & & & & 9 & 5 & 10 \\
\hline+ & + & & & & & 9 & 9 & 13 \\
\hline 208 & 20 & 9 & + & + & & $20 \overline{19}$ & 54 & 60 \\
\hline 9 & 2 & & & & & 136 & 14 & 15 \\
\hline
\end{tabular}

\begin{tabular}{|r|r|r|r|r|r|r|r|r|}
\hline 9 & 2 & & & & & 136 & 14 & 15 \\
\hline & & & & & & 110 & 33 & 40 \\
\hline 75 & 37 & 45 & + & 69 & & 1645 & 59 & 63 \\
\hline 113 & 200 & 13 & & 11 & 8 & 5777 & 64 & 66 \\
\hline 1 & 1 & & & + & & 93 & 40 & 57 \\
\hline+ & 3 & & & & & 62 & 25 & 36 \\
\hline & & 1 & & & & 32 & 16 & 18 \\
\hline
\end{tabular}

\begin{tabular}{|c|c|c|c|c|c|c|c|}
\hline 5 & 4 & 2 & 1 & 0 & 205 & 82 & \\
\hline 3 & 15 & 2 & 3 & 3 & 271 & 72 & 73 \\
\hline & & & & & 9 & 7 & 10 \\
\hline
\end{tabular}

\begin{tabular}{|r|r|r|r|r|r|r|r|r|}
\hline & & 2 & + & & & 29 & 15 & 21 \\
\hline & & & & & & 1205 & 16 & 17 \\
\hline 28 & 46 & 18 & 30 & 47 & 10 & 3629 & 93 & 95 \\
\hline & & 1 & 30 & 1 & 11 & 1520 & 58 & 61 \\
\hline 13 & 2 & 91 & 15 & 20 & 43 & 2510 & 90 & 91 \\
\hline & & & & & & 63 & 12 & 12 \\
\hline 2 & & & & & 1 & 195 & 23 & 24 \\
\hline 2 & & 11 & & 2 & 5 & 132 & 42 & 43 \\
\hline+ & & 2 & + & + & & 24 & 12 & 18 \\
\hline+ & & 80 & & + & 177 & 4886 & 51 & 57 \\
\hline & & & & + & 20 & 180 & 12 & 15 \\
\hline & & 2 & & 1 & & 41 & 23 & 31 \\
\hline+ & + & & & & & 240 & 25 & 27 \\
\hline 27 & & 1 & + & & 3 & 118 & 21 & 25 \\
\hline 1513 & 240 & 2 & 130 & 50 & & 12782 & 66 & 72 \\
\hline 1 & 3 & & & & & 11 & 9 & 11 \\
\hline & + & 8 & & & 1 & 46 & 15 & 18 \\
\hline+ & & 5 & + & 13 & & 1503 & 66 & 71 \\
\hline & & & & & & 426 & 15 & 17 \\
\hline 93 & 50 & 54 & 10 & 57 & 31 & 6534 & 88 & 90 \\
\hline 2 & & & & 6 & & 210 & 34 & 35 \\
\hline 1 & & & & & & 107 & 7 & 10 \\
\hline+ & & 13 & 75 & & & 2084 & 37 & 43 \\
\hline 773 & 200 & 52 & 10 & 160 & 250 & 18957 & 88 & 88 \\
\hline 27 & 13 & 24 & 10 & 15 & 17 & & & \\
\hline
\end{tabular}

\begin{tabular}{|r|r|r|r|r|r|}
\hline 27 & 13 & 24 & 10 & 15 & 17 \\
\hline 38 & 17 & 24 & 18 & 20 & 17 \\
\hline 9 & 0 & 2 & 1 & 0 & 1 \\
\hline 465 & 0 & 11 & 0 & 0 & 1 \\
\hline 3376 & 807 & 456 & 309 & 442 & 598 \\
\hline
\end{tabular}


Table 4. SPECIES SEEN ON NINE OR FEWER COUNTS

\begin{tabular}{|c|c|}
\hline SPECIES & LOCATION AND NUMBER \\
\hline \multirow{5}{*}{$\begin{array}{l}\text { COMMON LOON } \\
\text { YELLOW-BILLED LOON } \\
\text { AMERICAN WHITE PELICAN } \\
\text { TRUMPETER SWAN } \\
\text { CANADA GOOSE }\end{array}$} & Squaw Rapids, 1 \\
\hline & Gardiner Dam, 1 \\
\hline & Love-Torch River, 2 \\
\hline & Fort Qu'appelle, + \\
\hline & Endeavour, 1; Fort Qu'appelle, 1; Regina, 427; Squaw Rapids, 1 \\
\hline \multirow{5}{*}{$\begin{array}{l}\text { AM. GREEN-WINGED TEAL } \\
\text { LESSER SCAUP } \\
\text { HARLEQUIN DUCK } \\
\text { BUFFLEHEAD } \\
\text { COMMON MERGANSER }\end{array}$} & Fort Walsh, 1 \\
\hline & Fort Qu'appelle, 4; Gardiner Dam, 1 \\
\hline & Fort Qu'appelle, + \\
\hline & Fort Qu'appelle, +; Humboldt, +; Squaw Rapids, 1 \\
\hline & Gardiner Dam, 257; Indian Head, 2; Squaw Rapids, 5 \\
\hline \multirow{5}{*}{$\begin{array}{l}\text { TURKEY VULTURE } \\
\text { COOPER'S HAWK } \\
\text { RED-TAILED HAWK } \\
\text { ROUGH-LEGGED HAWK } \\
\text { PEREGRINE FALCON } \\
\text { GYREAI CON }\end{array}$} & Grayson, 2 \\
\hline & Indian Head, 1 \\
\hline & Regina, + \\
\hline & Glentworth, 2; Govenlock, 2; Grasslands Nat. Park, +; Qu'appelle V. Dam, 1 \\
\hline & $\begin{array}{l}\text { Crooked River, } 1 \\
\text { Duval +: Gardiner Dam 1. Hudson Bav + : Luseland } 1\end{array}$ \\
\hline & $\begin{array}{l}\text { Albert(B), } 1 ; \text {; Qu'appelle Valley Dam, } 1 ; \text { Weyburn, }+ \\
\text { Alba, } 1 \text {; Frince }\end{array}$ \\
\hline \multirow{2}{*}{$\begin{array}{l}\text { SPRUCE GROUSE } \\
\text { SAGE GROUSE }\end{array}$} & $\begin{array}{l}\text { Armit, 6; Big River, +; Meadow L., 6; Prince Albert National Park, +; } \\
\text { Squaw Rapids, } 4 \text {; Turtle Lake, } 2\end{array}$ \\
\hline & Ft. Walsh, +; Glentworth, 17; Govenlock, 14; Grasslands Nat. Park, + \\
\hline \multirow{5}{*}{$\begin{array}{l}\text { WILD TURKEY } \\
\text { MOURNING DOVE } \\
\text { BARRED OWL } \\
\text { LONG-EARED OWL } \\
\text { SHORT-EARED OWL }\end{array}$} & Fort Qu'appelle, 4; Fort Walsh, 6; Skull Creek, 1 \\
\hline & Edam, +; Glentworth, 1 ; Whitebeech, + \\
\hline & Squaw Rapids, + \\
\hline & Fort Walsh, 1 \\
\hline & $\begin{array}{l}\text { Glentworth, 2; Grasslands National Park, 1; Last Mountain Lake, } 1 \text {; } \\
\text { Moose Jaw, +; Raymore, }+; \text { Spring Valley, } 1 ; \text { Val Marie, } 1\end{array}$ \\
\hline \multirow{4}{*}{$\begin{array}{l}\text { BOREAL OWL } \\
\text { NORTHERN SAW-WHET OWL } \\
\text { RED-HEADED WOODPECKER } \\
\text { THREE-TOED WOODPECKER }\end{array}$} & Choiceland-Gronlid, 1 \\
\hline & Kamsack, + + \\
\hline & White Bear Lake, 1 \\
\hline & $\begin{array}{l}\text { Armit, 3; Candle Lake, 1; Choiceland-Gronlid, 1; Fort Walsh, +; } \\
\text { Hudson Bay, } 1 \text {; Somme, } 1 \text {; Squaw Rapids, } 2\end{array}$ \\
\hline \multirow{8}{*}{$\begin{array}{l}\text { NORTHERN FLICKER } \\
\text { AMERICAN CROW } \\
\text { BROWN CREEPER } \\
\text { GOLDEN-CROWNED KINGLET } \\
\text { TOWNSEND'S SOLITAIRE } \\
\text { VARIED THRUSH } \\
\text { BLACK-HEADED GROSBEAK } \\
\text { AMERICAN TREE SPARROW }\end{array}$} & $\begin{array}{l}\text { Ft. Qu'appelle, 4; Moose Jaw, 3; Pike L., 2; Prince Albert(B), +; Regina, } \\
\text { 10; Saskatoon, 12; Spinney Hill, 1; Swift Current, +; Weyburn, }+\end{array}$ \\
\hline & Fort Walsh, 1; Grenfell, 1; Moose Jaw, 1; Regina, 3; Saskatoon, 1 \\
\hline & Crooked Lake(B), 2; Fort Walsh, 1; Saskatoon, 2; Squaw Rapids, 1 \\
\hline & Ft. Walsh, 16; Raymore, +; Saskatoon, 1; Squaw Rapids, 3; Swift Current, + \\
\hline & Fort Walsh, 1 \\
\hline & Gardiner Dam, 1; Yorkton, 1 \\
\hline & Moose Jaw, 1 \\
\hline & $\begin{array}{l}\text { Big Muddy Lake, 8; Fort Walsh, 4; Glentworth, 3; Grasslands National } \\
\text { Park, 1; Raymore, } 1 \text { Saskatoon, 1; Skull Creek, 15; Weyburn, } 1\end{array}$ \\
\hline \multirow{5}{*}{$\begin{array}{l}\text { SAVANNAH SPARROW } \\
\text { FOX SPARROW } \\
\text { SONG SPARROW } \\
\text { WHITE-THROATED SPARROW } \\
\text { WHITE-CROWNED SPARROW } \\
\text { HARRIS' SPARROW }\end{array}$} & Saskatoon, 1 \\
\hline & Saskatoon, 1 \\
\hline & Fort Walsh, 2 \\
\hline & Saskatoon, 3; Spalding, 1; Spinney Hill, 1; Weyburn, 1 \\
\hline & Weyburn, 1 \\
\hline \multirow{4}{*}{$\begin{array}{l}\text { HARRIS' SPARROW } \\
\text { DARK-EYED JUNCO (Oregon) } \\
\text { DARK-EYED JUNCO (Pink-sided) } \\
\text { LAPLAND LONGSPUR }\end{array}$} & Indian Head, 1; Weyburn, 1 \\
\hline & Indian Head, +; Regina, 1 \\
\hline & Regina, 1 \\
\hline & $\begin{array}{l}\text { Big Muddy L., 26; Bromhead, 33; Glentworth, 365; Govenlock, 465; } \\
\text { Grasslands National Park, 42; Weyburn, } 458\end{array}$ \\
\hline \multirow{2}{*}{$\begin{array}{l}\text { CHESTNUT-COL. LONGSPUR } \\
\text { RED-WINGED BLACKBIRD }\end{array}$} & Govenlock, 1 \\
\hline & $\begin{array}{l}\text { Biggar, 1; Big Muddy Lake, 1; Fort Walsh, 5; Glentworth, 3; } \\
\text { Grasslands National Park, 37; Last Mountain Lake W.M.U., } 1 \\
\end{array}$ \\
\hline BREWER'S BLACKBIRD & $\begin{array}{l}\text { Biggar, } 1 \text {; Grasslands National Park, } 2 \text {; Hepburn, 1; Last Mountain L. } \\
\text { W.M.U., 1; Love-Torch River, 2; Regina, 1; Swift Current, 6: Warman, } 1\end{array}$ \\
\hline \multirow{5}{*}{$\begin{array}{l}\text { COMMON GRACKLE } \\
\text { BROWN-HEADED COWBIRD } \\
\text { ROSY FINCH } \\
\text { PURPL FINCH } \\
\text { RED CROSSBILL }\end{array}$} & Grasslands National Park, 1; Kamsack, 1 ; Regina, 11; Weyburn, 1 \\
\hline & Glentworth, 1; Grasslands National Park, 2 \\
\hline & Glentworth, 7; Meadow Lake, 2; Skull Creek, 38 \\
\hline & Kamsack, 18; MacDowall, +; Round L, +; Switt Curent, 3; Weybum, 2 White Bear L, 10 \\
\hline & Ft. Walsh, 67; Livelong, +; Saskatoon, 5; Squaw Rapids, 3; Switt Current, + \\
\hline
\end{tabular}




\begin{tabular}{|l|l|}
\hline SPECIES & LOCATION AND NUMBER \\
\hline EAGLE species & Swift Current, + \\
\cline { 2 - 2 } WOODPECKER species & Armit, 3; Clark's Crossing, 1; Squaw Rapids, 1 \\
\cline { 2 - 2 } REDPOLL species & $\begin{array}{l}\text { Biggar, 15; Craven, 8; Fort Walsh, 24; Govenlock, 2; Regina, 2; } \\
\text { Squaw Rapids, 38 }\end{array}$ \\
\cline { 2 - 2 } & Fort Walsh, 20; Saskatoon, 7 \\
\hline
\end{tabular}

Table 6. SUMMARY OF NEW OR TYING HIGH COUNTS ESTABLISHED DURING THE 1991 COUNTS (tying counts regular type; boldface indicates a new record).

\begin{tabular}{|c|c|c|c|c|}
\hline LOCATION & $\begin{array}{r}1991 \\
\text { COUNT }\end{array}$ & SPECIES & $\begin{array}{l}\text { IOUS } \\
\mathrm{HIGH}\end{array}$ & LOCATION AND YEAR \\
\hline Squaw Rapids & 1 & Common Loon & 1 & $\begin{array}{l}\text { Gardiner Dam (82-84); Grand Centre } \\
\text {-Pierceland (87); Squaw Rapids (89) }\end{array}$ \\
\hline Gardiner Dam & 1 & Yellow-billed Loon & & NEW \\
\hline $\begin{array}{l}\text { Love-Torch River } \\
\text { Fort Qu'Appelle }\end{array}$ & $\begin{array}{l}2 \\
1\end{array}$ & $\begin{array}{l}\text { Am. White Pelican } \\
\text { Hariequin Duck }\end{array}$ & $\begin{array}{l}2 \\
1\end{array}$ & $\begin{array}{l}\text { Regina (59) } \\
\text { Gardiner Dam (90) }\end{array}$ \\
\hline Grayson & 2 & Turkey Vulture & 1 & Fort Walsh (84); Edam (90) \\
\hline Indian & 1 & Cooper's Hawk & 1 & 8 counts north to $N$. Battleford \\
\hline River & 1 & Peregrine Falcon & 1 & 17 counts $N$ to Squaw Rapids - Carrot R. \\
\hline Armit; Crooked R. & 4 & Northern Hawk Owl & 2 & $\begin{array}{l}\text { Masefield (66); Battleford-N. Battle- } \\
\text { ford (77); Squaw Rapids-Sipanok (79) }\end{array}$ \\
\hline Choiceland-Gronlid & 1 & Boreal Owl & 1 & 18 counts $s$. to Indian Head \\
\hline Kams & 1 & Northern Saw-whet Owl & 1 & 33 counts $n$. to Maidstone Bridge \\
\hline White Bear Lake & 1 & Red-headed Woodpecker & & NEW \\
\hline $\begin{array}{l}\text { Saskatoon } \\
\text { Saskatoon }\end{array}$ & $\begin{array}{l}37 \\
36\end{array}$ & $\begin{array}{l}\text { Downy Woodpecker } \\
\text { Hairy Woodpecker }\end{array}$ & $\begin{array}{l}32 \\
28\end{array}$ & $\begin{array}{l}\text { Saskatoon }(90) \\
\text { Saskatoon }(90)\end{array}$ \\
\hline $\begin{array}{l}\text { Saskatoon } \\
\text { Hudson Bay }\end{array}$ & $\begin{array}{r}86 \\
261\end{array}$ & $\begin{array}{l}\text { Blue Jay } \\
\text { Common Raven }\end{array}$ & $\begin{array}{r}66 \\
205\end{array}$ & $\begin{array}{l}\text { Togo (77) } \\
\text { Prince Albert (89) }\end{array}$ \\
\hline Saskatoon & 390 & Black-capped Chickadee & 285 & Saskatoon (90) \\
\hline Gardiner Dam & 1 & Varied Thrush & 1 & 5 counts $n$. to Pierce Lake \\
\hline Fort Walsh & 6 & Northern Shrike & 6 & $\begin{array}{l}\text { Saskatoon (63); Pike Lale (69); } \\
\text { Ft. Walsh }(89,90)\end{array}$ \\
\hline Moose Jaw & 1 & Black-headed Grosbeak & & NEW \\
\hline Saskatoon & 1 & Fox Sparrow & 1 & Moose Jaw (85); Biggar (87) \\
\hline Saskatoon & 1 & Savannah Sparrow & & NEW \\
\hline Fort Walsh & 2 & Song Sparrow & 2 & Ft. Qu'Appelle (88); Ft. Walsh (90) \\
\hline Weyburn & 1 & White-crowned Sparrow & 1 & $\begin{array}{l}\text { Saskatoon (74); Fort Walsh (80); } \\
\text { Broadview (86) }\end{array}$ \\
\hline lock & 1 & Chestnut-collared Longsp & & NEW \\
\hline ands I & 37 & Red-winged Blackbird & 30 & Estevan (62) \\
\hline White Bear & 3 & Western Meadowlark & 3 & Skull Creek (71) \\
\hline $\begin{array}{l}\text { Regina } \\
\text { Kamsack }\end{array}$ & $\begin{array}{l}11 \\
18\end{array}$ & $\begin{array}{l}\text { Common Grackle } \\
\text { Purple Finch }\end{array}$ & $\begin{array}{r}3 \\
14\end{array}$ & $\begin{array}{l}\text { Regina (67); Weyburn (87) } \\
\text { Saskatoon (69) }\end{array}$ \\
\hline
\end{tabular}

